\title{
DISTRIBUIÇÃo GEOGRÁFICA DAS PTERIDÓFITAS OCORRENTES NO ESTADO DE PERNAMBUCO, BRASIL
}

\author{
Iva Carneiro Leão Barros ${ }^{1}$ \\ Osvaldo Carneiro de Lira ${ }^{1}$ \\ Albanita de Jesus R. da Silva ${ }^{2}$
}

Recebido em 15-10-86. Aceito em 14-8-88.

\begin{abstract}
RESUMO - Apresenta-se um levantamento das espécies de pteridófitas coletadas em cerca de 55 municípios do Estado de Pernambuco, visando ao estudo da distribuição da flora pteridofítica pelas várias zonas fisiográficas. Com este trabalho, aumentou-se o número de espécies de pteridófitas para o Estado, de 121 referidas anteriormente, para 194 espécies, com a freqüência e a dispersão por cada zona fitogeográfica. Das 194 espécies encontradas, três ocorrem na zona do litoral, nenhuma sendo exclusiva desta zona; 162 na subzona de mata úmida, sendo 96 exclusivas da área; 37 na subzona de mata seca, sendo quatro exclusivas da área; 65 na subzona de mata serrana ou brejos de altitude, sendo 16 exclusivas da área; oito na zona das savanas (tabuleiros), ocorrentes em outras zonas fisiográficas; e 26 nas diferentes zonas de caatinga do agreste e sertão. Destas, a espécie Selaginella sellowii Hieron é exclusiva da zona fisiográfica das caatingas, embora Selaginella convoluta (Arn.) Spring. apresente sua maior ocorrência nessa zona. As espécies se distribuem pelas famílias Ophioglossaceae, Marattiaceae, Schizaeaceae, Gleicheniaceae, Hymenophyllaceae, Cyatheaceae, Pteridaceae, Vittariaceae, Dennstaedtiaceae, Thelypteridaceae, Dryopteridaceae, Aspleniaceae, Davalliaceae, Blechnaceae, Polypodiaceae, Marsileaceae, Salviniaceae, Psilotaceae, Lycopodiaceae e Selaginellaceae. A espécie de maior distribuição é Anemia tomentosa (Sav.) Sw., ocorrendo desde a subzona de mata úmida até a subzona de caatinga do sertão do Jatinã.
\end{abstract}

Palavras-chave: distribuição, pteridófitas, PE-Brasil.

\begin{abstract}
A survey of the species of Pteridophytes which were collected in about 55 municipalities in the State of Pernambuco was made in order to study the distribution of the pteridophytic flora over several physiographic zones. Through this study, the number of species of Pteridophytes was increased in the State. One hundred and twenty-one species were referred by Pontual. Now, 194 species were presented, including Pontual's species, with information about frequency and dispersion per phytogeographical zones. From those 194 species which were found, 3 occur in the littoral zone (none of the species are limited to this zone); 162 species were refer-
\end{abstract}

\footnotetext{
${ }^{1}$ Profs. Adjuntos, Departamento de Botânica, Centro de Ciências Biológicas, UFPE - Av. Prof. Artur de Sá, s/no - Cidade Universitária - Recife-PE

${ }^{2}$ Estagiária, Departamento de Botânica/CCB/ Bolsista do CNPq, Processo n? 109387/84 - Curso de Mestrado em Criptógamos - UFPE
} 
red to humid forest subzone with 96 species limited to this area; 37 species to dry forest subzone with 4 species limited to this third area; 65 species to sierra forest subzone or "brejo" of altitude with 16 species limited to this fourth area. Eight species were referred to savannah zone ("tabuleiros") and 26 species occurred in different zones of the "caatinga" of "sertão". Selaginella sellowii Hieron is limited to this area and Selaginella convoluta (Arn.) Spring. is very frequent in this area. The species are distributed among the following families: Ophioglossaceae, Marattiaceae, Schizaeaceae, Gleicheniaceae, Hymenophyllaceae, Cyatheaceae, Pteridaceae, Vittariaceae, Dennstaedtiaceae, Thelypteridaceae, Dryopteridaceae, Aspleniaceae, Davalliaceae, Blechnaceae, Polypodiaceae, Marsileaceae, Salviniaceae, Psilotaceae, Lycopodiaceae and Selaginellaceae. Anemia tomentosa (Sav.) Sw. shows the widest distribution. It can be found from humid forest subzone of "caatinga" of "sertão do Jatinã".

Key words: distribution, Pteridophytes, PE-Brasil.

\section{Introdução}

O Estado de Pernambuco, localizado na região Nordeste do Brasil, apresenta diferentes zonas fitogeográficas, que segundo Andrade-Lima (1960) são classificadas em zonas do litoral, da mata, da caatinga e das savanas. Em todas estas zonas fitogeográficas há uma representativa flora pteridofítica.

Alguns autores têm trabalhado com a flora de pteridófitas de Pernambuco, como Barros (1980, 1982 e 1982a), Barros \& Mariz (1985), Barros et al. (1986 e 1986a), Barros et al. (1985 e 1986) e Pontual (1969, 1971 e 1972). Outros trabalhos, como os de Andrade-Lima (1950, 1960, 1961 e 1969), Sarmento (1960) e Sehnem $(1968,1968$ a , 1970, 1971, 1972, 1974 e 1979) referem pteridófitas para Pernambuco, em seus textos, embora não sejam específicos para o assunto. Este é o primeiro trabalho abordando a distribuição geográfica das pteridófitas no Estado, abrangendo o estudo da situação das espécies por municípios, nas diversas regiões fisiográficas, listando as espécies mais freqüentes e mais bem distribuídas, evidenciando a freqüência das espécies mais representativas e representando no mapa do Estado de Pernambuco os percentuais de ocorrência das famílias por regiões fisiográficas.

\section{Material e métodos}

As espécies de pteridófitas incluídas neste trabalho são coletas dos autores e de outros pesquisadores cujas exsicatas foram depositadas nos herbários UFP, HST, PEUFR e IPA, perfazendo um total de 546 amostras estudadas, tendo-se adotado a ordenação filogenética dos táxons de Tryon \& Tryon (1982). Quando necessário, utilizou-se a técnica de diafanização de Strittmater (1973) para a observação do padrão de venação na identificação de algumas espécies.

A conceituação das várias zonas fisiográficas do Estado é a de Andrade-Lima (1960). 


\section{Resultados e comentários}

Os resultados são apresentados em tabelas, discriminando a ocorrência das espécies por municípios que compõem as zonas e subzonas fitogeográficas do Estado de Pernambuco. A visualização destas zonas e subzonas é evidenciada no mapa fitogeográfico (Fig. 1), ao qual estão associados gráficos representativos do percentual de ocorrência das famílias de pteridófitas.

Uma análise ecológica mais aprofundada sobre a ocor ^ância das pteridófitas nestas áreas fitogeográficas, elaborada pelos autores deste trabalho, está em fase de conclusão.

Seguindo a classificação fisiográfica de Andrade-Lima (1960), as espécies foram ordenadas segundo: a) zona do litoral; b) zona da mata; c) zona das savanas; d) zona das caatingas.

\section{a) Zona do litoral}

A zona do litoral se caracteriza por uma flora bem definida que habita a faixa do litoral, sob a influência direta ou indireta do mar, não é uniforme em todas as suas características e apresenta uma largura variável, que vai de poucos metros até alguns quilômetros. Tem sua localização ao longo da costa atlântica, e, na subzona das restingas e terraços litorâneos, vamos ter a ocorrência, nos campos de restinga, das espécies Blechnum serrulatum Rich. e Pteridium aquilinum (L.) Kuhn e, nos mangues, a espécie Acrostichum aureum L., segundo referências de AndradeLima (1960). Esta zona do litoral apresenta altitude, tipo de solo e maior ou menor concentração salina característicos e que se fazem traduzir por variações sensíveis na vegetação, que é então subdividida em subzonas de flora própria e fisionomia bem definida.

\section{b) Zona da mata}

A zona da mata se faz representar como o ponto de ligação das florestas orientais brasileiras, que vêm do sul, com as florestas equatoriais brasileiras, vindas da Amazônia (Andrade-Lima, 1960). É representada, segundo a ocorrência de pteridófitas em nosso Estado, pela subzona de mata úmida, subzona de mata seca e subzona de mata serrana (brejos de altitude). A subzona de mata úmida, onde foi realizada a maioria das coletas, é representada por matas bastante úmidas, cortadas por riachos, cachoeiras e grandes açudes, ambientes propícios à ocorrência das pteridófitas. A vegetação é exuberante, perenifólia e rica em cipós.

Subzona de mata úmida - As coletas de pteridófitas na subzona de mata úmida sobressaem com um maior número de espécies nos municípios de:

a) Cabo - cerca de 39 espécies, havendo uma predominância das Pteridaceae, seguidas das Schizaceae com seis espécies;

b) Escada - dominância para o grupo das Polypodiaceae com seis espécies, sendo referidas para o município 32 espécies distribuídas por 10 famílias; 
c) Recife - resquício da Floresta Atlântica (Mata de Dois Irmãos) na área metropolitana, onde se referem 33 espécies, com uma presença significativa das Pteridaceae com nove espécies;

d) Rio Formoso - a maioria da espécies foi coletada na Estação Florestal Experimental (EFLEX) de Saltinho, sob controle do IBDF, havendo também coletas realizadas em córregos próximos a essa área. Há uma predominância das Polypodiaceae com quatro espécies, sendo referidas para o município 20 espécies;

e) Tapera - ocorrência de 53 espécies, com 21 Pteridaceae e seis Davalliaceae.

f) Quipapá - a riqueza de espécies é evidente, com a presença de 63 espécies, sendo a flora de pteridófitas muito rica, comparando-se com outros municípios em semelhantes condições ambientais.

g) São Lourenço da Mata - registro de 33 espécies, com predominância das Pteridaceae com 10 espécies e das Polypodiaceae com oito.

Algumas espécies são raras ou pobres em suas ocorrências, como: Danaea elliptica J.E. Smith, referida apenas para Rio Formoso; Ophioglossum reticulatum L., referida para o Cabo e Rio Formoso; Psilotum nudum (L.) Beauv., referida para Dois Irmãos e Quipapá; Diplazium ambiguum Raddi., Diplazium brasiliensis Ros., Lomagramma guianensis (Aubl.) Ching e Asplenium lunulatum Sw., referidas apenas para Rio Formoso. Isto talvez seja devido à pobreza de coletas, mas também à provável dispersão restrita dessas espécies em Pernambuco.

Nos municípios de mata úmida constatou-se uma grande representatividade das famílias Pteridaceae, ocorrendo com 46 espécies, Dryopteridaceae com 33 e Polypodiaceae com 27 espécies. Com menor representatividade estão as Schizaeaceae, ocorrendo com 11 espécies, as Selaginellaceae com nove, as Davalliaceae com oito, as Aspleniaceae e Dennstaedtiaceae com sete e as demais com número abaixo de cinco espécies, sendo consideradas pobres quanto à ocorrência.

A subzona de mata seca caracterizada por uma menor exuberância da vegetação, motivada pela menor umidade ambiental, variação de altitude, permeabilidade do solo e maior proximidade da zona de caatinga, é caducifólia e apresenta um maior número de indivíduos arbóreos por área, com caules longos e número de cipós vigorosos menor (Andrade-Lima, 1960).

As coletas de pteridófitas nessa subzona de mata seca se representam por seis municípios, sobressaindo com uma maior distribuição o Município de Vicência, com 29 espécies, ocorrendo 14 da família Pteridaceae, a mais bem representada, seguida da Polypodiaceae com seis espécies.

No cômputo geral, temos 37 espécies ocorrendo nas matas secas de Pernambuco, representadas pelas Schizaeaceae, Hymenophyllaceae, Cyatheaceae, Pteridaceae, Vittariaceae, Dennstaedtiaceae, Thelypteridaceae, Dryopteridaceae, Aspleniaceae, Blechnaceae e Polypodiaceae. Sobressaem as Pteridaceae, ocorrendo com 16 espécies para a zona fisiográfica de mata seca.

Os gêneros mais representativos são Adiantum Linn. e Polypodium Linn. 
TABELA 1 - Subzona de mata úmida

FAMILIAS: OPHIOGLOSSACEAE/MARATTIACEAE/SCHIZAEACEAE/GLEICHENIACEAE/ HYMENOPHYLLACEAE/CYATHEACEAE

\begin{tabular}{|c|c|c|c|c|c|c|c|c|c|c|c|c|c|}
\hline ESPECIES & $M U N I C I P I O S$ & 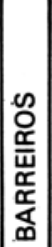 & $\begin{array}{l}0 \\
\infty \\
\varangle \\
0\end{array}$ & 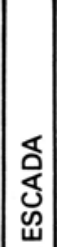 & $\begin{array}{l}\nwarrow \\
\swarrow \\
\text { 어 }\end{array}$ & 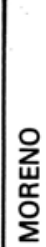 & 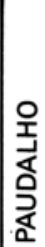 & $\frac{\mathbb{6}}{\frac{0}{2}}$ & 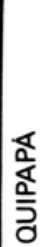 & 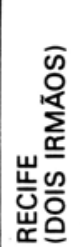 & 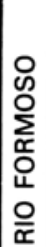 & 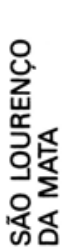 & 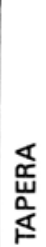 \\
\hline
\end{tabular}

\section{OPHIOGLOSSACEAE}

Ophioglossum reticulatum L.

MARATTIACEAE

Danaea eliptica J.E. Smith

\section{SCHIZAEACEAE}

Lygodium volubile Sw.

Lygodium venustum Sw.

Schizaea pennula (Hook.) Sw.

Anemia hirta (L.) Sw.

Anemia phyllitidis (L.) Sw.

Anemia hirsuta (L.) Sw.

Anemia pastinacaria Moritz. ex Prantl.

$\times$

Anemia ciliata PresI.

Anemia tomentosa (Sav.) Sw. var.

tomentosa Sw.

Anemia tomentosa (Sav.) Sw. var. anthriscifolia (Schrad.) Mickel

Anemia flexuosa (Sav.) Sw.

\section{GLEICHENIACEAE}

Dicranopteris pectinata (Willd.) Underw.

Dicranopteris flexuosa (Schrad.) Underw.

HYMENOPHYLLACEAE

Trichomanes pinnatum Hedw.

Trichomanes ovale (Fourn.) W. Boer.

Trichomanes kraussii Hook. \& Grév.

\section{CYATHEACEAE}

Trichipteris microdonta (Desv.) Tryon

Trichipteris procera (Willd.) Tryon

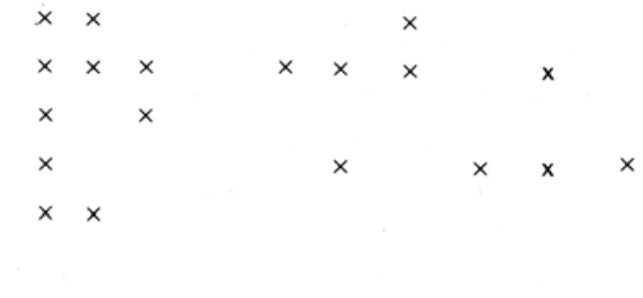

$\times$

$x \quad \times$

X

$x \quad \times$

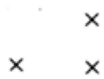

$\times$

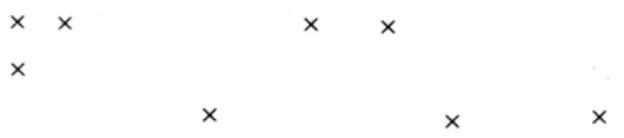

$\times$
$\times$


TABELA 1 - Subzona de mata úmida

FAMILIAS: PTERIDACEAE/VITTARIACEAE (continuação)

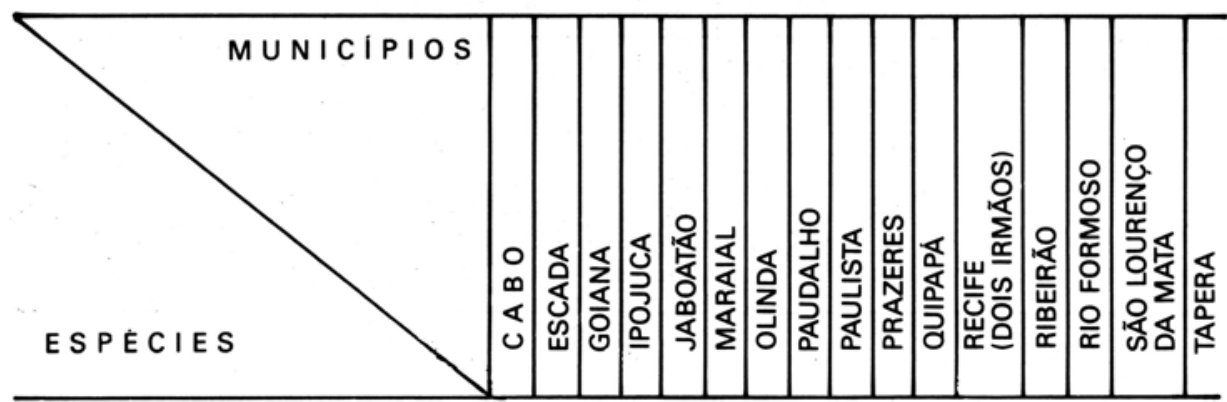

PTERIDACEAE

Adiantum obliquum Willd.

$\times$

Adiantum villosum $\mathrm{L}$.

Adiantum terminatum Kze.

Adiantum petiolatum Desv.

Adiantum pectinatum Ettingsh.

Adiantum trapeziforme $\mathrm{L}$.

Adiantum pentadactylon Langsd.

\& Fisch.

Adiantum glaucescens Klotz.

Adiantum capillus-veneris $\mathrm{L}$.

Pteris denticulata Sw.

Pteris leptophyllum Sw.

Pteris biaurita L.

Pteris longifolia L.

Pteris vittata $\mathrm{L}$.

Pteris kunzeana Ag.

Pteris brasiliensis Raddi.

Acrostichum aureum L.

Acrostichum danaeifolium Langsd.

$\&$ Fisch.

\section{VITTARIACEAE}

Vittaria lineata (L.) Sm.

Vittaria graminifolia Kaulf.

Anetium citrifolium (L.) Splitg.

Ananthacorus angustifolius (Sw.)

Underw. \& Maxon.

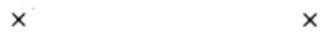

$\times$

$\times$

$\times$

$\times$

$\times$

$\times \quad \times$

$\times$

$\times$

$\times \quad \times$

$\times$

$\times$ 
TABELA 1 - Subzona de mata úmida

FAMILIA: DENNSTAEDTIACEAE / PTERIDACEAE (continuação)

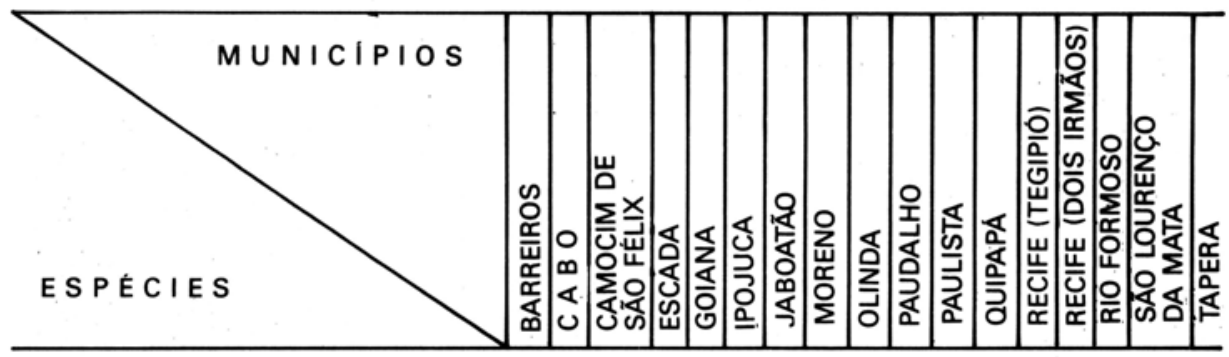

DENNSTAEDTIACEAE

Microlepia speluncae (L.) Moore

Sacoloma elegans Kaulf.

Pteridium aquilinum (L.) Kuhn
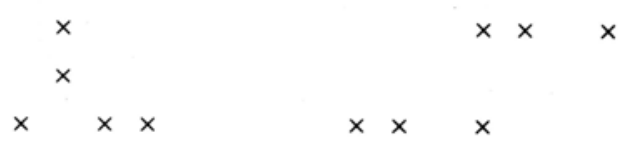

\section{PTERIDACEAE}

Pityrograma calomelanos (L.) L.

Adiantopsis radiata (L.) Fée

Adiantopsis pedata (Hook.) Moore

Hemionitis tomentosa (Lam.) Raddi. $\times$

Hemionitis palmata L.

Ceratopteris thalictroides (L.) Brong. $\times$

Cheilanthes concolor (Langsd. \&

Fisch.) R. \& A. Tryon

Doryopteris collina (Raddi.) J. Sm.

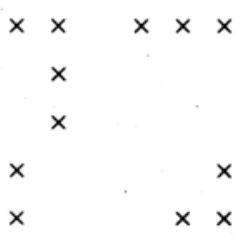

Doryopteris pedata (L.) Fée var. pedata Fée

Doryopteris pedata (L.) Fée var.

multipartida (Fée) Tryon

Doryopteris ludens (Wall.) J. Sm.

Doryopteris sagittifolia (Raddi.)

J. Sm.

Doryopteris varians (Raddi.) J. Sm.

Adiantum tenerum Sw.

Adiantum deflectens Mart.

Adiantum diagoanum Glaz.

Adiantum dolosum Kze.

$\times$

Adiantum fovearum Raddi.

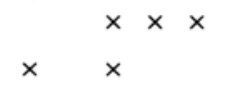


TABELA 1 - Subzona de mata úmida

FAMILIAS: PTERIDACEAE/DENNSTAEDTIACEAE/THELYPTERIDACEAE (continuação)

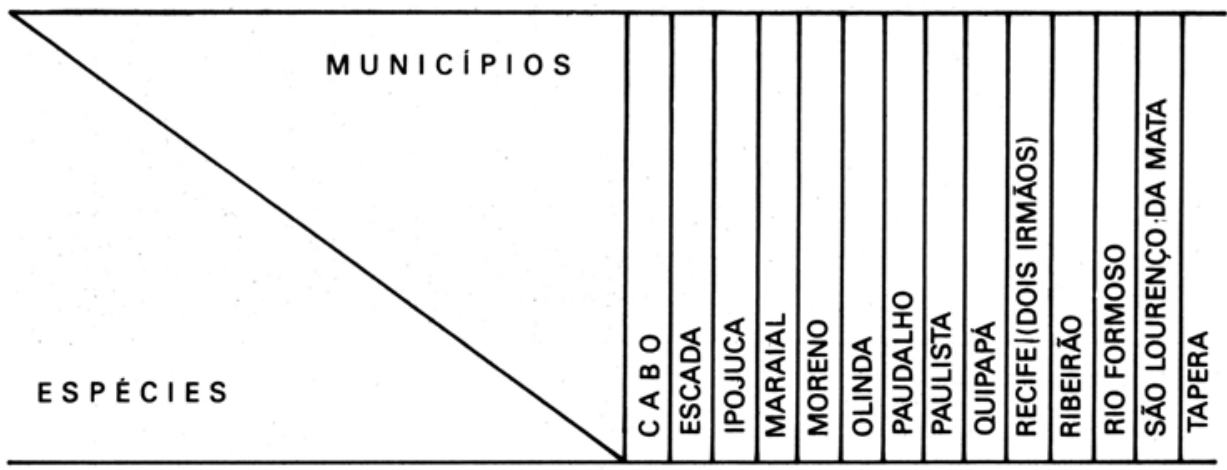

PTERIDACEAE

Adiantum latifolium Lam.

Adiantum intermedium Sw.

Adiantum lunulatum Burm.

Adiantum lucidum Sw.

Adiantum serratodentatum Willd.

Adiantum tetraphyllum Willd.

Adiantum pulverulentum $\mathrm{L}$.

Adiantum caudatum L.

Adiantum sordidum Lind.

\section{DENNSTAEDTIACEAE}

Lindsaea guianensis (Aubl.) Dryand.

Lindsaea quadriangularis Raddi.

Lindsaea stricta (Sw.) Dryand.

Lindsaea lancea (L.) Bedd.

Lindsaea trapeziforme Dryand.

\section{THELYPTERIDACEAE}

Thelypteris dentata (Forsk.) E.P. St. John

Thelypteris reticulata (L.) Proctor

Thelypteris serrata (Cav.) Alston

Thelypteris totta (Thumb.) Schelpe
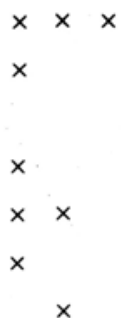

$\times$

$\times$

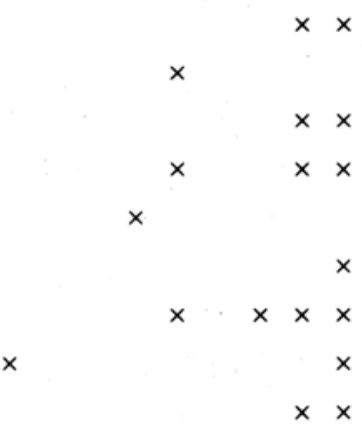

$\times$

$\times$

$\times$

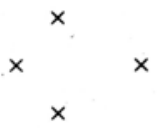

$\times$

$\times x \times$

$\times \times \times$

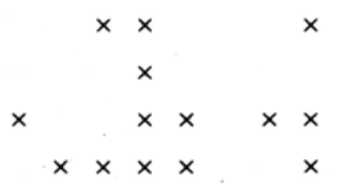


TABELA 1 - Subzona de mata úmida

FAMILIA: DRYOPTERIDACEAE (continuação)

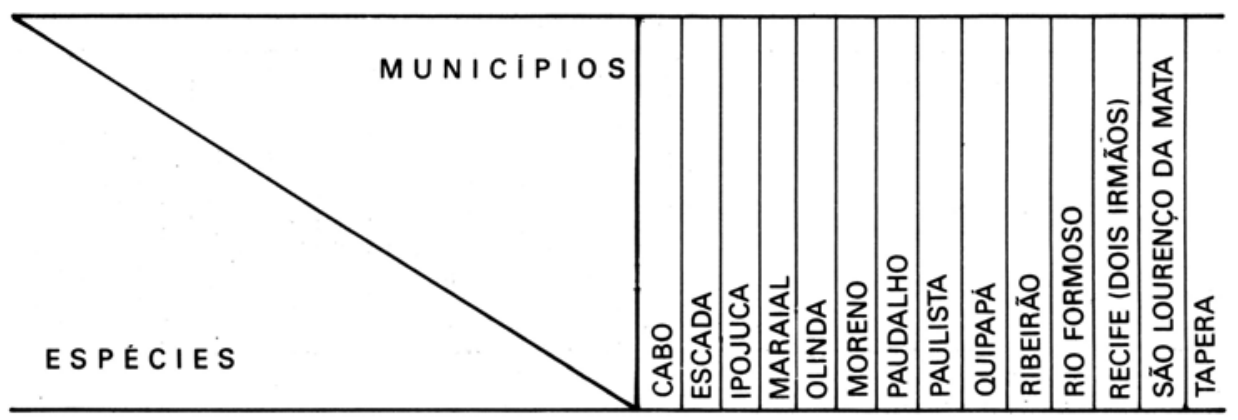

\section{DRYOPTERIDACEAE}

Tectaria heracleifolia (Willd). Underw.

Tectaria incisa Cav.

$\times$

Rumohra adiantiformis (Forst.) Ching

Dryopteris normalis C. Chr.

$\times$

Dryopteris paucijuga C. Chr.

$\times$

Dryopteris setigera (BI.) O. Ktze.

Dryopteris ctenitis (Link.) O. Ktze.

Dryopteris alsophilacea (Kunze) O. Ktze.

Dryopteris mollis (Jacq.) Hieron

$\times$

Dryopteris effusa (Sw.) Urb.

Stigmatopteris santi-gabrielli (Hook.) C. Chr.

Didymochlaena lunulata Desv.

Didymochlaena trunculata (Sw.) J. Sm.

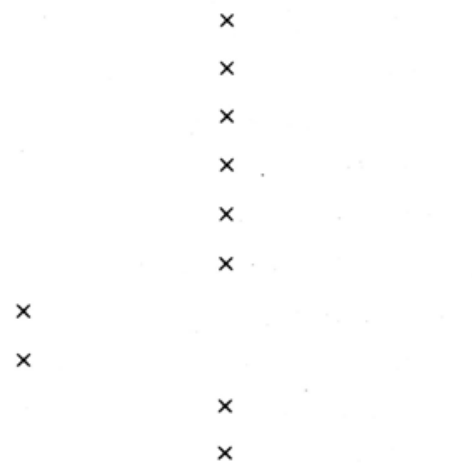

Polybotrya cervina (L.) Kep.

Polybotrya serratifolia (Fée) KI.

Diplazium cristatum (Desr.) Alston

Diplazium plantaginifolium Urban

continua... 
TABELA 1 - Subzona de mata úmida

FAMILIAS: DRYOPTERIDACEAE/ASPLENIACEAE/DAVALLIACEAE/BLECHNACEAE (continuação)

ESPECIES MUNICIPIOS

DRYOPTERIDACEAE

Diplazium ambiguum Raddi.

Diplazium brasiliensis Ros.

Diplazium shepherdii (Spring.) Link.

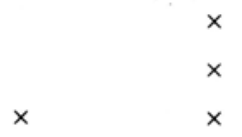

Lomariopsis sorbifolia (L.) Fée

Lomagrama guianensis (Aubl.) Ching

ASPLENIACEAE

Asplenium formosum Willd.

Asplenium lunulatum Sw.

Asplenium claussenii Hieron

Asplenium otites Ling.

Asplenium brachyotus Kze.

Asplenium serratum L.

Asplenium cristatum Lam.

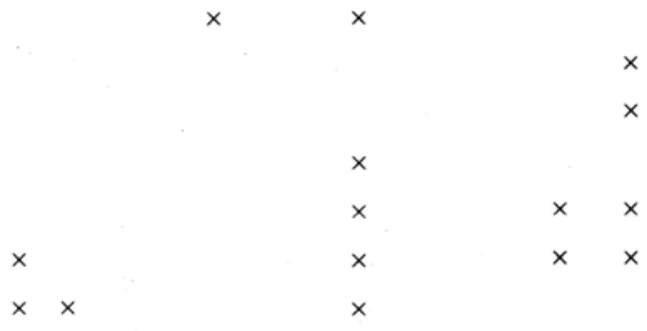

DAVALLIACEAE

Nephrolepis exaltata (L.) Schott.

Nephrolepis pickelli Ros.

$\times$

$\times \quad \times$

Nephrolepis pectinata (Willd.) Schott.

Nephrolepis hirsutula (Forst.) Presl.

Nephrolepis bisserrata (Sw.) Schott.

Nephrolepis duffii Hort.

Nephrolepis cordifolia (L.) Presl.

\section{BLECHNACEAE}

Blechnum occidentale L.

Blechnum brasiliense Desv.

Blechnum serrulatum L.C. Rich.

Salpichlaena volubilis (Kaulf.) Hook.

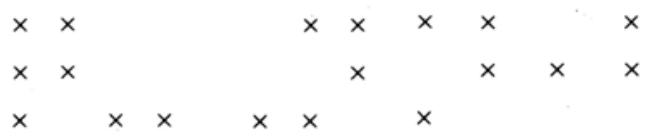

continua... 
TABELA 1 - Subzona de mata úmida

FAMILIA: POLYPODIACEAE (continuação)

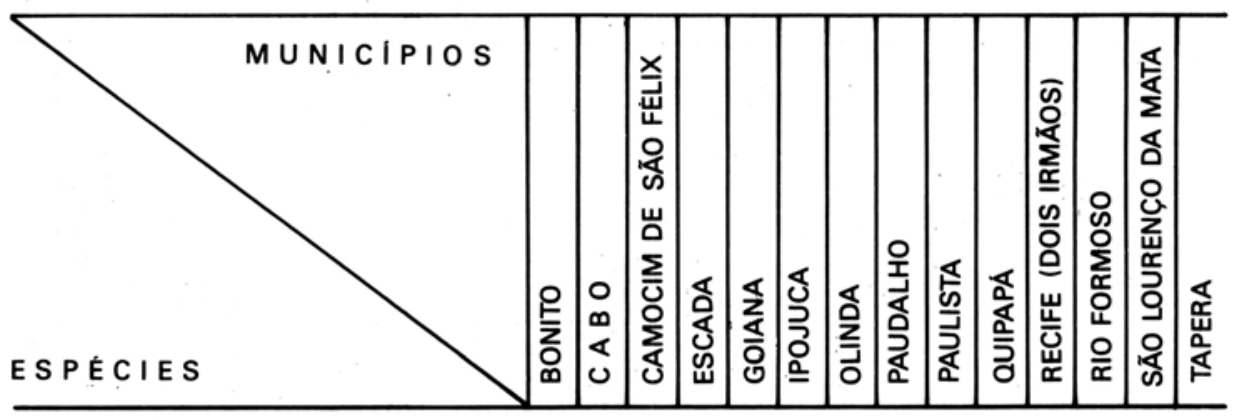

POLYPODIACEAE

Dicranoglossum furcatum (L.)

J. Sm.

Dicranoglossum desvauxii (KI.)

Proctor

Micrograma vaccinifolia

(Langsd. \& Fisch.) Copel.

Micrograma geminata

(Schrad.) R. \& A. Tryon

Pleopeltis macrocarpa

(Willd.) Kaulf.

Polypodium brasiliense Poir.

Polypodium aureum L.

Polypodium decumanum Willd.

Polypodium hirsutissima Raddi.

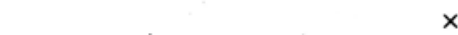

Polypodium catharinae

(Langsd.) \& Fisch.

Polypodium lapathifolium Poiret.

$\times$

Polypodium escorpioides $\mathrm{L}$.

Polypodium stenophyllum BI.

Polypodium lineatum Col.

Polypodium lineare Thumbr.

Polypodium lycopodioides L.

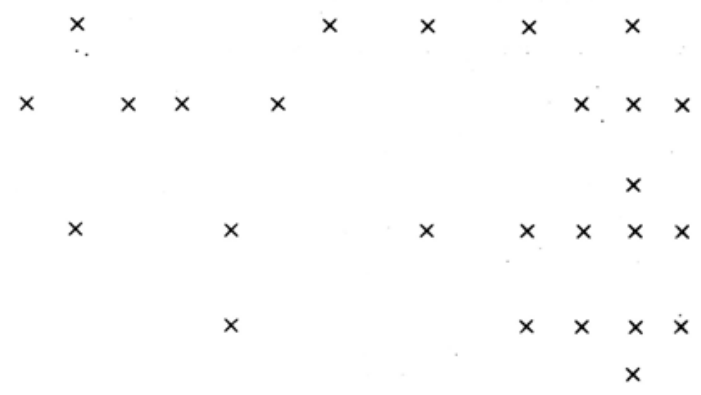

Pleopeltis revoluta

(Willd.) A.R. Sm.

Polypodium fraxinifolium Jacq.

$\times$

Polypodium crassifolium L.

Polypodium areolatum H.B.W.

Polypodium laevigatum Cav. 
TABELA 1 - Subzona de mata úmida

FAMILIAS: POLYPODIACEAE/MARSILEACEAE/SALVINIACEAE/PSILOTACEAE/

LYCOPODIACEAE/SELAGINELLACEAE (conclusão)

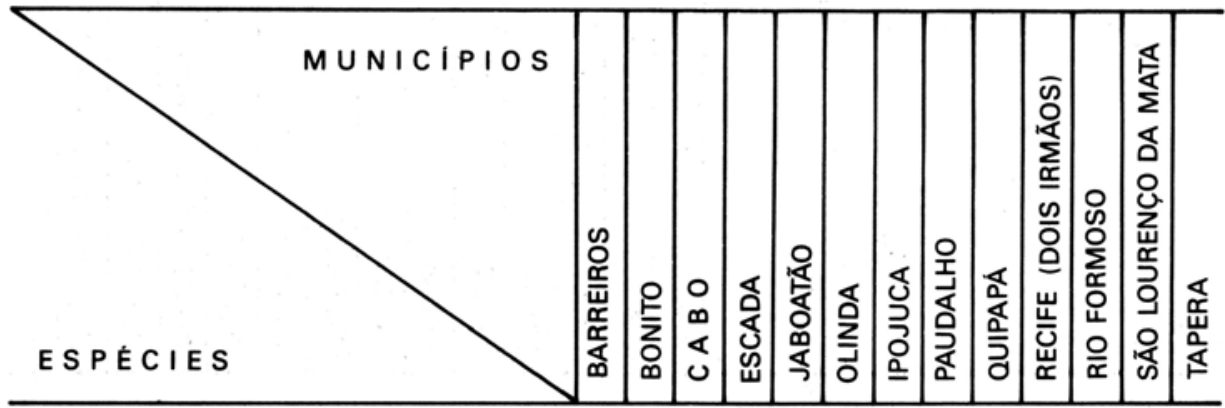

POLYPODIACEAE

Polypodium pectinatum $\mathrm{L}$.

Polypodium pectinatiforme Lind.

Campyloneurum repens (Aubl.) Presl.

Campyloneurum transparens Ett.

Campyloneurum angustifolium (Sw.) Fée $\times$

MARSILEACÉAE

Marsilea deflexa A. Braum

$\times$

SALVINIACEAE

Salvinia auriculata Aubl.

Azolla caroliniana Willd.

PSILOTACEAE

Psilotum nudum (L.) Beauv.

LYCOPODIACEAE

Lycopodium cernuum L.

Lycopodium heterocarpon Fée

Lycopodium carolinianum L.

SELAGINELLACEAE

Selaginella convoluta (Arn.) Spring.

Selaginella breynei Spring. ex Char.

Selaginella willdenowi (Desv.) Baker

Selaginella simplex Baker

Selaginella muscova $\mathrm{A}$. Br.

Selaginella flagellata Spring.

Selaginella deltoides A. Br.

Selaginella fragillima A. Silv.

$\times \quad \times$

Selaginella vallida Alston 
TABELA 2 - Subzona de mata seca

FAMILIAS: SCHIZAEACEAE/HYMENOPHYLLACEAE/CYATHEACEAE/PTERIDACEAE

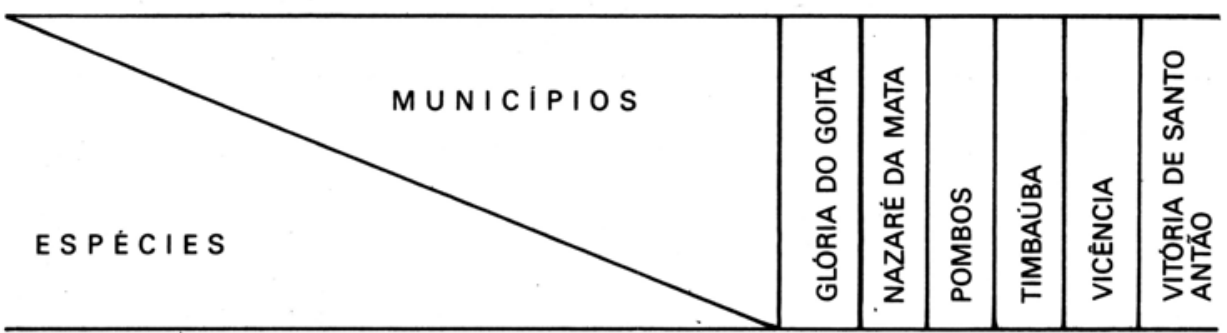

\section{SCHIZAEACEAE}

Anemia hirta (L.) Sw.

Anemia phyllitidis (L.) Sw.

Lygodium venustum Sw.

$\times$

$\times$

$\times$

Schizaea elegans (Vahl.) Sw.

$\times$

HYMENOPHYLLACEAE

Trichomanes pinnatum Hedw.

Trichomanes kraussii Hook. \& Grév.

CYATHEACEAE

Trichipteris microdonta (Desv.) Tryon

PTERIDACEAE

Adiantum fovearum Raddi.

Adiantum intermedium Sw.

Adiantum lunulatum Burm.

Adiantum pulverulentum $\mathrm{L}$.

Adiantum cuneatum Langsd. \& Fisch.

Adiantum obliquum Willd.

Adiantopsis radiata (L.) Fée var. froesii Brade

Adiantopsis radiata (L.) Fée var. radiata Fée

Adiantopsis pedata (HK.) Moore

$\times$

Pteris leptophyllum Sw.

Pteris denticulata Sw.

Doryopteris pedata (L.) Fée var. pedata Fée

Doryopteris pedata (L.) Fée var. multipartida (Fée) Tryon

Hemionitis palmata L.

Acrostichum daneaefolium Langsd. \& Fisch.

$\times$ 
TABELA 2 - Subzona de mata seca

FAMILIAS: VITTARIACEAE/DENNSTAEDTIACEAE/THELYPTERIDACEAE DRYOPTERIDACEAE/ASPLENIACEAE/BLECHNACEAE/POLYPODIACEAE (conclusão)

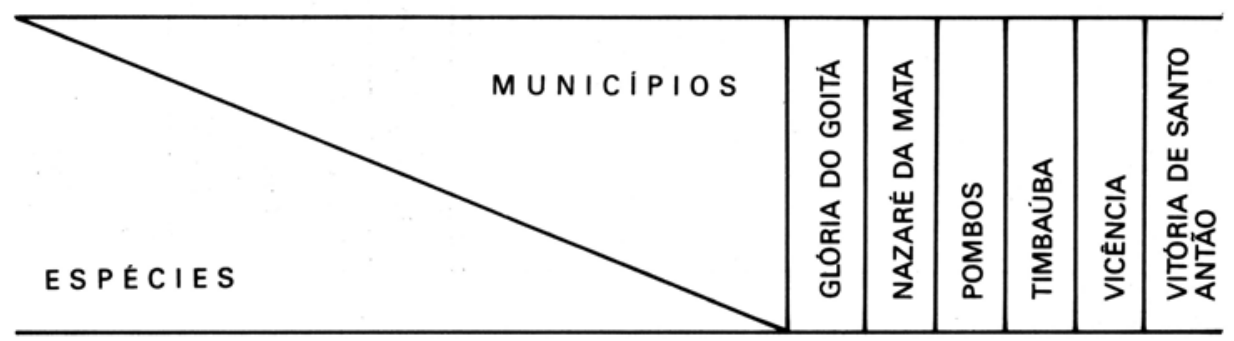

VITTARIACEAE

Vittaria lineata (L.) Sm. $\times$ DENNSTAEDTIACEAE

Lindsaea stricta (Sw.) Dryand.

Microlepia speluncae (L.) Moore

HELYPTERIDACEAE

Thelypteris serrata (Cav.) Alston

DRYOPTERIDACEAE

Dryopteris normalis C. Chr.

Stigmatopteris santi-gabrielli (Hook.) C. Chr. ASPLENIACEAE

Asplenium serratum L.

\section{BLECHNACEAE}

Blechnum occidentale L.

Blechnum brasiliense Desv.

POLYPODIACEAE

Polypodium phyllitidis L.

Polypodium landigii L.

Polypodium lineatum Col.

Polypodium fraxinifolium Jacq.

$\times$

Pleopeltis revoluta (Willd.) A.R. Sm.

Micrograma geminata (Schrad.) R. \& A. Tryon

Campyloneurum repens (Aublet.) Presl.

$\times$
$\times$
$\times$
$\times$

Campyloneurum angustifolium (Sw.) Fée 
TABELA 3 - Subzona de mata serrana (brejos)

FAMILIAS: SCHIZAEACEAE/CYATHEACEAE/PTERIDACEAE

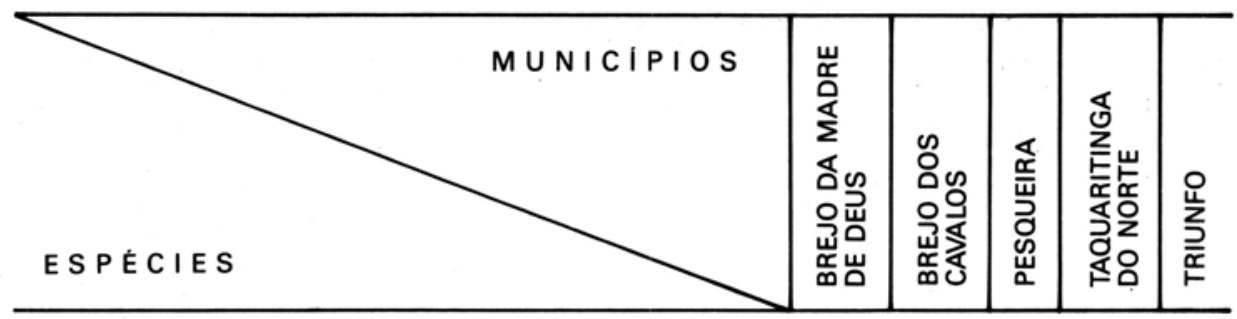

\section{SCHIZAEACEAE}

Anemia filiformis (Sav.) Sw.

Anemia hirta (L.) Sw.

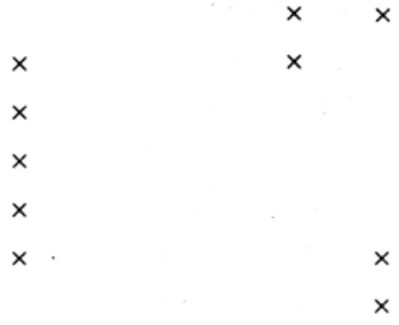

Anemia flexuosa (Sav.) Sw.

Anemia oblongifolia (Cav.) Sw.

Anemia phyllitidis (L.) Sw.

Anemia villosa H.B.

Anemia mirabilis Brade

Anemia tomentosa (Sav.)

Sw. var. anthriscifolia (Schrad.) Mickel

Schizaea elegans (Vahl.) Sw.

CYATHEACEAE

Cyathea delgadii Sternb.

$\times$

\section{PTERIDACEAE}

Adiantum cuneatum Langsd. \& Fisch.

Adiantum obliquum Willd.

Adiantum brasiliense Raddi.

Adiantum pulverulentum $\mathrm{L}$.

Adiantopsis radiata (L.) Fée

Doryopteris pedata (L.) Fée var. pedata Fée

Doryopteris collina (Raddi.) J. Sm.

Cheilantes concolor (Langsd. \& Fisch.) R. \& A. Tryon

Pteris biaurita L.

Pteris denticulata Sw.

Pteris dissimilis (Fée) Chr.

Hemionitis tomentosa (Lam.) Raddi. $\times$

$\times$

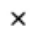

$\times$

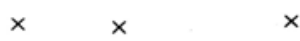

$\times$

$\times$

$\times$

$\times$

$\times \quad \times$

$\times \quad \times$ 
TABELA 3 - Subzona de mata serrana (brejos)

FAMILIAS: VITTARIACEAE/DENNSTAEDTIACEAE/THELYPTERIDACEAE/ DRYOPTERIDACEAE/ ASPLENIACEAE/DAVALLIACEAE/BLECHNACEAE/ POLYPODIACEAE (continuação)

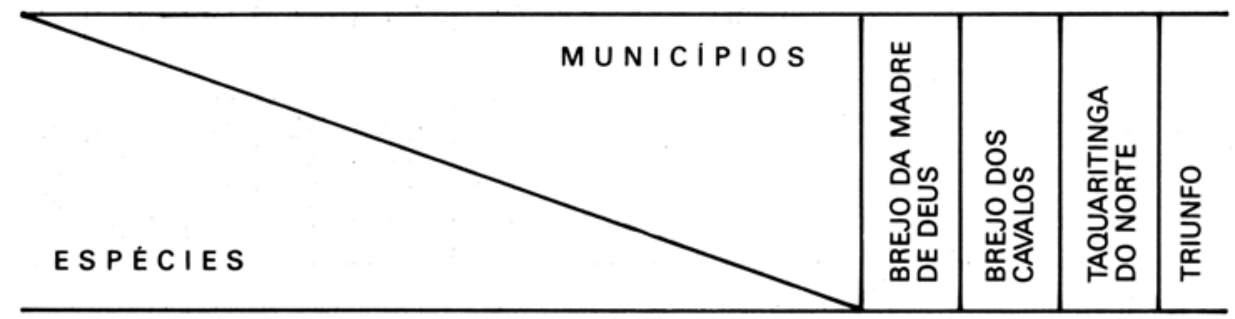

VITTARIACEAE

Vittaria lineata (L.) Sm.

$\times$

\section{DENNSTAEDTIACEAE}

Lindsaea ovoidea Fée.

Lindsaea stricta (Sw.) Dryand.

$\times$

Lindsaea trapeziforme Dryand.

$\times$

Lindsaea lancea (L.) Bedd.

Pteridium aquilinum (L.) Kuhn

$\times$

$\times$

THELYPTERIDACEAE

Thelypteris dentata (Forsk.) E.P. St. John

$\times$ DRYOPTERIDACEAE

Rumohra adiantiformis (Forst.) Ching

Polybotrya scandens (Raddi.) Christ.

Lomariopsis sorbifolia (L.) Fée

$\times$

$\times$

ASPLENIACEAE

Asplenium formosum Willd.

Asplenium claussenii Hieron

$\times$

$\times$

DAVALLIACEAE

Nephrolepis bisserrata (Sw.) Schott.

\section{BLECHNACEAE}

Blechnum brasiliense Desv.

Blechnum occidentale L.

POLYPODIACEAE

Polypodium fraxinifolium Jacq.

Polypodium brasiliense Poir.

Polypodium hirsutissimum Raddi. 
TABELA 3 - Subzona de mata serrana (brejos)

FAMILIAS: POLYPODIACEAE/LYCOPODIACEAE (conclusão)

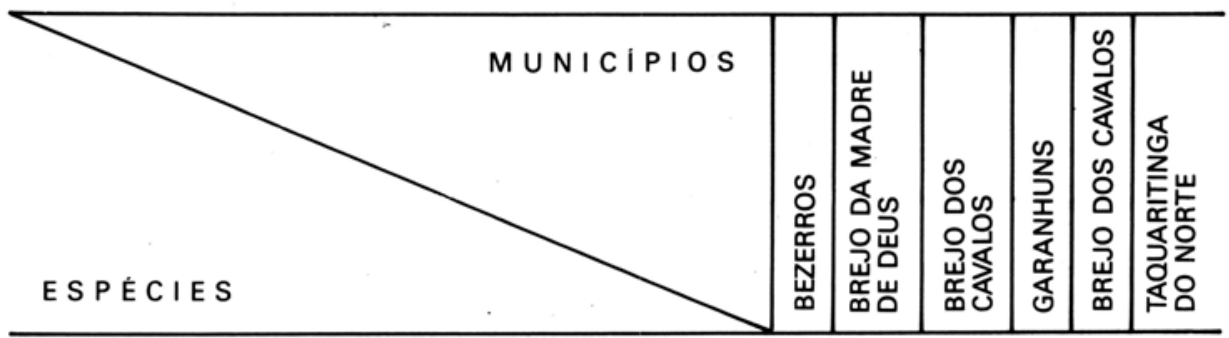

POLYPODIACEAE

Polypodium glofium L.

$\times$

Polypodium polypodioides (L.) Watt.

Polypodium apiculatum Kze.

Polypodium angustum (H.B.W.) Liebm.

Polypodium areolatum H.B.W.

Polypodium catharinae Langsd. \& Fisch.

Polypodium aureum L.

Polypodium paradiseae Langsd. \& Fisch.

Polypodium lepidopteris (Langsd. \& Fisch.) Kze.

Polypodium stenophyllum BI.

Pleopeltis revoluta (Willd.) A.R. Sm.

Micrograma geminata (Schrad.) R. \& A. Tryon

Dicranoglossum furcatum (L.) J. Sm.

Dicranoglossum desvauxii (KI.) Proctor

Cochlidium paucinervatum (Fée) C. Chr.

$\times$

$\times$

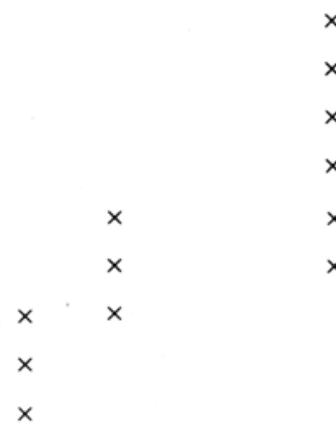

LYCOPODIACEAE

Lycopodium cernuum L.

Lycopodium taxifolium L.

Lycopodium setaceus (Hamilt.) Hert.

$\times$

Lycopodium heterocarpon Fée

$\times$

$\times$

Lycopodium martii Alv. Silv. 
TABELA 4 - Zona das savanas (tabuleiros)

FAMILIAS: SCHIZAEACEAE/DENNSTAEDTIACEAE/THELYPTERIDACEAE/BLECHNACEAE/ POLYPODIACEAE

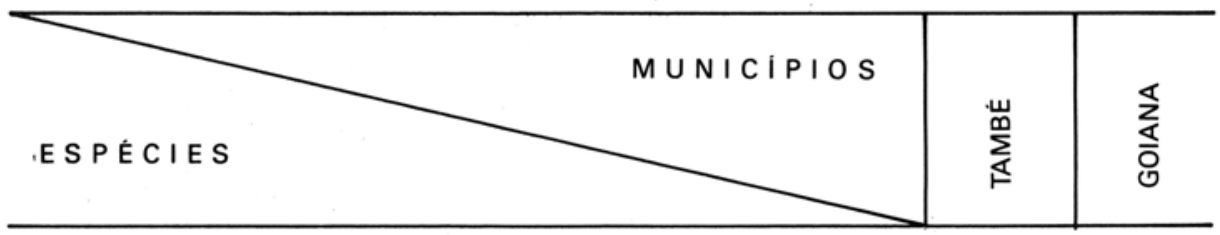

SCHIZAEACEAE

Schizaea pennula (Hook.) Swartz.

$\times \quad x$

Lygodium venustum Sw.

$\times$

DENNSTAEDTIACEAE

Pteridium aquilinum (L.) Kuhn

$\times$

$\times$ THELYPTERIDACEAE

Thelypteris totta (Thunb.) Schelpe

Thelypteris serrata (Cav.) Alston

BLECHNACEAE

Blechnum serrulatum L.C. Rich.

POLYPODIACEAE

Polypodium decumanum Willd.

Polypodium stenophyllum BI. 
A subzona de mata serrana ou brejos de altitude se faz representar por matas perenifólias, que encimam muitas das serras dos três quartos ocidentais do Estado. Apresenta cotas de pluviosidade nunca inferiores a $500 \mathrm{~m}$ e até $1.100 \mathrm{~m}$, onde ocorrem variações de umidade e temperatura durante os períodos chuvoso e de estio, determinando condições especiais que resultam em uma facies própria dessas florestas serranas com espécies típicas e fisionomia distinta (Andrade-Lima, 1960).

As coletas de pteridófitas nessa subzona foram mais freqüentes em cinco matas de brejo de altitude.

Há uma representatividade significativa de espécies para as matas serranas ou brejos de altitude, com um total de 65 espécies. Um predomínio das Polypodiaceae com 18 espécies, seguidas das Pteridaceae com 12 e das Schizaeaceae com nove.

As coletas em áreas de matas serranas ou brejos foram realizadas em sete municípios diferentes, sobressaindo-se, em número de espécies, Taquaritinga do Norte com 36 e Brejo da Madre de Deus com 22 espécies.

Quanto à mata serrana de Taquaritinga do Norte, hoje praticamente toda devastada e ocupada por cafezal, é válido ressaltar que se refere para essa área uma flora pteridofítica bastante especial, com a ocorrência de cinco espécies de Polypodium, uma de Cochlidium, cinco de Lycopodium e uma de Rumohra, que foram coletadas apenas nessa área.

Para o Brejo da Madre de Deus, tem-se uma espécie de Lindsaea e uma de Polybotrya, que são referidas apenas para essa área.

\section{c) Zona das savanas}

A zona das savanas (tabuleiros) se caracteriza por uma vegetação com um manto herbáceo, com predominância de gramíneas, intercaladas por arvoretas tortuosas com súber espesso e folhas pouco coriáceas. É subdividida em duas subzonas, onde, na subzona dos tabuleiros, vamos encontrar a ocorrência de oito espécies de pteridófitas.

Com respeito às áreas de tabuleiros do Estado de Pernambuco, observam-se apenas duas manchas dessa fisionomia vegetacional (Andrade-Lima, 1960), uma em També e a outra em Goiana. Não há predominância de nenhuma família, nem maior representatividade de um gênero sobre outros.

\section{d) Zona das caatingas}

A zona das caatingas é a maior das zonas fitogeográficas do Estado, caracterizando-se por uma vegetação de porte médio a baixo, tropófila (decídua), rica em espinhos, com muitos representantes das Cactáceas e Bromeliáceas, com um clima seco e o solo em grande parte raso. A pluviosidade, fatores do solo e a altitude condicionam a variabilidade do aspecto vegetacional das caatingas (Andrade-Lima, 1960). São encontrados representantes das pteridófitas tanto na subzona do agreste como na subzona do sertão, nas regiões do sertão central, ser- 
TABELA 5 - Subzona das caatingas

FAMILIAS: SCHIZAEACEAE/CYATHEACEAE/PTERIDACEAE

\begin{tabular}{|c|c|c|c|c|c|c|c|c|c|c|c|c|c|c|c|c|c|c|}
\hline \multirow{2}{*}{$\begin{array}{l}\text { CARACTERIZACCÃO } \\
\text { DAS SUBZONAS } \\
\text { DAS CAATINGAS } \\
\text { MUNICIPIOS }\end{array}$} & \multicolumn{5}{|c|}{$\begin{array}{l}\text { SUBZONA DE } \\
\text { CAATINGA DO } \\
\text { AGRESTE }\end{array}$} & \multicolumn{2}{|c|}{$\begin{array}{c}\text { SUBZONA } \\
\text { DE } \\
\text { CAATINGA } \\
\text { DO SERTÁO } \\
\text { DO SÃO } \\
\text { FRANCISCO }\end{array}$} & \multicolumn{7}{|c|}{$\begin{array}{l}\text { SUBZONA DE CAATINGA DO } \\
\text { SERTAOO CENTRAL }\end{array}$} & \multicolumn{2}{|c|}{\begin{tabular}{|} 
SUBZONA \\
DE \\
CAATINGA \\
DO SERTÃO \\
DOS \\
CHAPADOES \\
ARENITICOS
\end{tabular}} & $\begin{array}{c}\text { SUBZONA } \\
\text { DE } \\
\text { CAATINGA } \\
\text { DO SERTÃO } \\
\text { DO JATINA } \\
\end{array}$ & $\begin{array}{l}\text { SUBZO- } \\
\text { NA DE } \\
\text { CAATIN- } \\
\text { GA DO } \\
\text { SERTÃO } \\
\text { DO } \\
\text { ARA- } \\
\text { RIPE }\end{array}$ \\
\hline & $\begin{array}{l}\frac{x}{1} \\
\frac{1}{2} \\
\\
0 \\
\\
\end{array}$ & 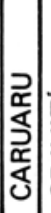 & 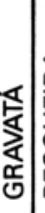 & 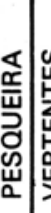 & 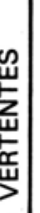 & 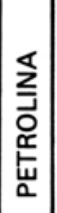 & 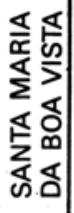 & 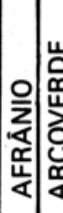 & כ) & 峁 & 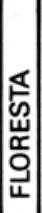 & 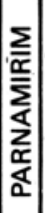 & 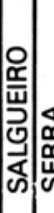 & 选选 & $\begin{array}{l}u \\
2 \\
0 \\
0 \\
\partial \\
0\end{array}$ & $\begin{array}{l}\varangle \\
2 \\
\varangle \\
z \\
-\end{array}$ & 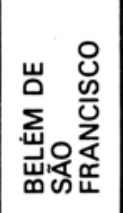 & 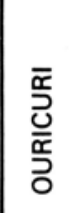 \\
\hline
\end{tabular}

\section{SCHIZAEACEAE}

Anemia hirta Sw.

Anemia villosa H.B.

Anemia tomentosa

(Sav.) Sw. var.

tomentosa Sw.

Anemia tomentosa

(Sav.) Sw. var.

anthriscifolia

(Schrad.) Mickel

Anemia oblongifolia

(Cav.) Sw.

Anemia filiformis

(Sav.) Sw.

Lygodium venustum

Sw.

CYATHEACEAE

Alsophila praecincta

Kze. .

PTERIDACEAE

Pityrograma

calomelanos (L.)

Link.

Doryopteris varians

(Raddi.) J. Sm. 
TABELA 5 - Subzona das caatingas

FAMILIAS: PTERIODACEAE/DRYOPTERIDACEAE/BLECHNACEAE/POLYPODIACEAE (continuação)

\begin{tabular}{|c|c|c|c|c|c|c|c|c|c|c|c|c|c|c|c|c|c|c|}
\hline $\begin{array}{l}\text { CARACTERIZAC̄ÃO } \\
\text { DAS SUBZONAS } \\
\text { DAS CAATINGAS }\end{array}$ & \multicolumn{5}{|c|}{$\begin{array}{l}\text { SUBZONA DE } \\
\text { CAATINGA DO } \\
\text { AGRESTE }\end{array}$} & \multicolumn{2}{|c|}{\begin{tabular}{|c|} 
SUBZONA \\
DE \\
CAATINGA \\
DO SERTÁO \\
DO SÃO \\
FRANCISCO
\end{tabular}} & \multicolumn{7}{|c|}{$\begin{array}{l}\text { SUBZONA DE CAATINGA DO } \\
\text { SERTÃO CENTRAL }\end{array}$} & \multicolumn{2}{|c|}{\begin{tabular}{|c|} 
SUBZONA \\
DE \\
CAATINGA \\
DO SERTAOO \\
DOS \\
CHAPADOES \\
ARENITICOS
\end{tabular}} & $\begin{array}{c}\text { SUBZONA } \\
\text { DE } \\
\text { CAATINGA } \\
\text { DO SERTAOO } \\
\text { DO JATINA } \\
\end{array}$ & $\begin{array}{l}\text { SUBZO- } \\
\text { NA DE } \\
\text { CAATIN- } \\
\text { GA DO } \\
\text { SERTAOO } \\
\text { DO } \\
\text { ARARI- } \\
\text { PE }\end{array}$ \\
\hline ESPECIES & 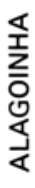 & 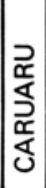 & 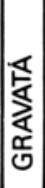 & 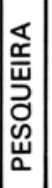 & 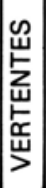 & 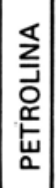 & 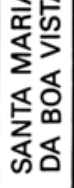 & 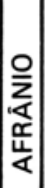 & 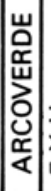 & 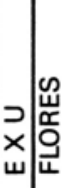 & 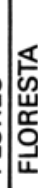 & 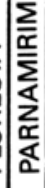 & ○्ञ & 我造 & $\begin{array}{l}w \\
\partial \\
0 \\
\frac{0}{\partial} \\
\infty\end{array}$ & $\begin{array}{l}\varangle \\
\square \\
\varangle \\
z\end{array}$ & 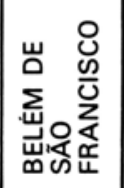 & $\begin{array}{l}\overline{\bar{x}} \\
\frac{\mathcal{U}}{\tilde{\sigma}} \\
\frac{0}{0}\end{array}$ \\
\hline
\end{tabular}

Hemionitis

tomentosa (Lam.)

Raddi.

DRYOPTERIDACEAE

\section{Stigmatopteris \\ meniscioides \\ (Willd.) Kramer \\ BLECHNACEAE \\ Blechnum \\ occidentale $\mathrm{L}$.}

POLYPODIACEAE

Pleopeltis revoluta

(Willd.) A.R. Smith

Polypodium

crassifolium L.

Polypodium

brasiliense Poir.

Polypodium

polypodioides (L.)

Watt.

Polypodium

hirsutissimum

Raddi.

Dicranoglossum

furcatum (L.)

J. Sm. 
TABELA 5 - Subzona das caatingas

FAMILIAS: MARSILEACEAE/SALVINIACEAE/LYCOPODIACEAE/SELAGINELLACEAE (conclusão)

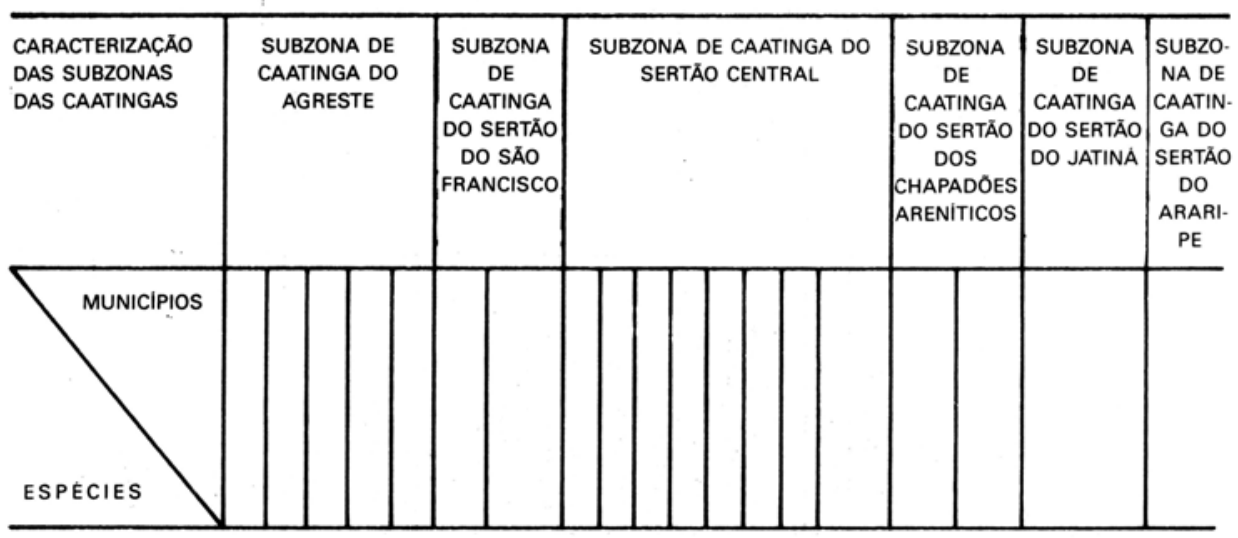

MARSILEACEAE

Marsilea quadrifolia

L.

SALVINIACEAE

\section{Azolla caroliniana}

Willd.

Azolla microphylla

Kaulf.

LYCOPODIACEAE

Lycopodium

cernuum $\mathrm{L}$.

Lycopodium

linifolium $\mathrm{L}$.

SELAGINELLACEAE

Selaginella

convoluta (Arn.)

Spring.

Selaginella

sellowii Hieron 
tão dos chapadões areníticos, sertão do São Francisco, sertão do Jatinã e sertão do Araripe.

\section{Subzona de caatinga do agreste}

Verifica-se a ocorrência de nove famílias de pteridófitas, representadas por 18 espécies distribuídas por vários municípios da região de caatinga do agreste.

As pteridófitas nessa região, onde o clima de semi-aridez já se faz significativo, não são de ampla dispersão, contudo estão presentes em toda a região.

Subzona de caatinga do sertão do São Francisco

Observa-se a presença mais evidente das Schizaeaceae, ocorrendo com quatro espécies, dispersas pelos municípios de Petrolina e Santa Maria da Boa Vista, na região de caatinga do sertão do São Francisco. Além das quatro espécies do gênero Anemia Swartz, temos apenas nessa área a ocorrência de Selaginella convoluta (Arn.) Spring., que se faz bastante presente por todas as regiões do sertão de Pernambuco.

\section{Subzona de caatinga do sertão central}

Evidencia-se, no tocante à diferenciação das zonas de caatinga, uma maior representatividade de espécies nas subzona de caatinga do sertão central, com a ocorrência de nove espécies das famílias Schizaeaceae, Pteridaceae, Polypodiaceae, Salviniaceae e Selaginellaceae. Não há predominância de nenhum grupo sobre outro, nos oito municípios onde há registro de coletas.

\section{Subzona de caatinga do sertão dos chapadões areníticos}

Observa-se, para a subzona de caatinga do sertão dos chapadões areníticos, a ocorrência de quatro espécies de pteridófitas. Poder-se-ia pensar nas manchas de vegetação de mata serrana dessas áreas, mas as observações referidas nos exemplares são para locais secos esporadicamente inundáveis.

A presença de Polypodium polypodioides (L.) Watt. nessa região, referida também para Taquaritinga do Norte, deixa sugerida a possibilidade de evidente altitude na área de coleta.

Subzona de caatinga do sertão do Jatinã

Vê-se pela Tabela 5 , com referência à subzona de caatinga do sertão do Jatinã, a presença apenas das Schizaeaceae, com a espécie Anemia tomentosa (Sav.) Sw. var. tomentosa Sw., sugerindo-nos uma grande aridez ambiental, que parece não propiciar a dispersão de outras pteridófitas na área.

\section{Subzona de caatinga do sertão do Araripe}

Foram encontradas nas áreas inundáveis do Município de Ouricuri (Silva, 1958) as espécies Selaginella convoluta (Arn.) Spring., Azolla caroliniana L. e Marsilea quadrifolia $\mathrm{L}$. A presença de pteridófitas aquáticas pelos sertões nordestinos nos períodos chuvosos é um fato bastante comum observado pelos pesquisadores que 
TABELA6 - Distribuição geral das pteridófitas no Estado de Pernambuco, segundo as zonas fisiográficas FAMILIAS: OPHIOGLOSSACEAE/MARATTIACEAE/SCHIZAEACEAE (continuação)

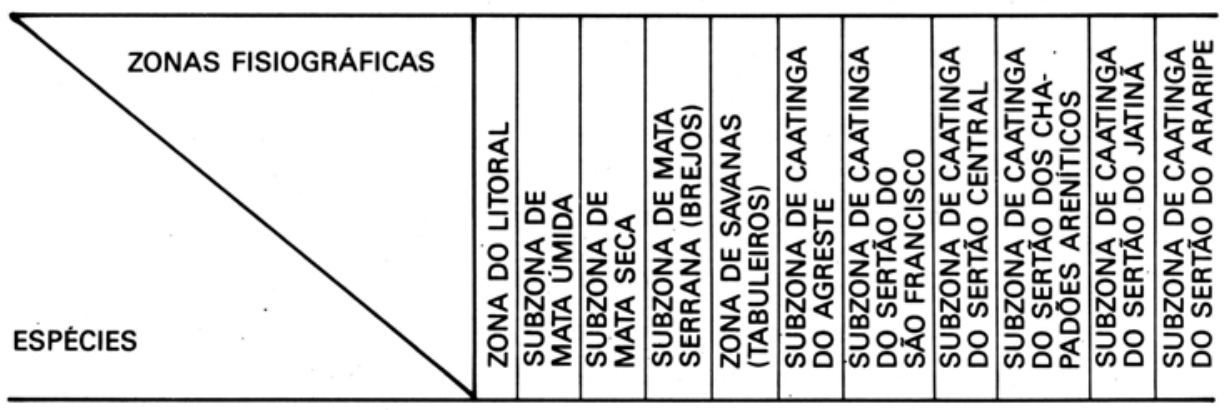

OPHIOGLOSSACEAE

Ophioglossum reticulatum L.

\section{MARATTIACEAE}

Danaea eliptica J.E. Smith

\section{SCHIZAEACEAE}

Lygodium volubile Sw.

Lygodium venustum $\mathrm{Sw}$.

Schizaea pennula (Hook.) Sw.

Schizaea elegans (Vahl.) Sw.

Anemia filiformis (Sav.) Sw.

Anemia hirta (L.) Sw.

Anemia phyllitidis (L.) Sw.

Anemia oblongifolia (Cav.) Sw.

Anemia hirsuta (L.) Sw.

Anemia pastinacaria Moritz. ex

Prantl.

Anemia ciliata Presl.

Anemia tomentosa (Sav.) Sw. var.

tomentosa Sw.

Anemia tomentosa (Sav.) Sw. var.

anthriscifolia (Schrad.) Mickel

Anemia flexuosa (Sav.) Sw.

Anemia villosa H.B.

Anemia mirabilis Brade

$$
\begin{array}{lllllllllll}
- & \times & - & - & - & - & - & - & - & - & - \\
- & \times & \times & - & \times & \times & - & - & - & - & - \\
- & \times & - & - & \times & - & - & - & - & - & - \\
- & - & \times & \times & - & - & - & - & - & - & - \\
- & - & - & \times & - & - & \times & \times & - & - & - \\
- & \times & \times & \times & - & \times & - & - & - & - & - \\
- & \times & \times & \times & - & - & - & - & - & - & - \\
- & - & - & \times & - & - & \times & - & - & - & - \\
- & \times & - & - & - & - & - & - & - & - & - \\
& & & & & & & & & & \\
- & \times & - & - & - & - & - & - & - & - & - \\
- & \times & - & - & - & - & - & - & - & - & -
\end{array}
$$


TABELA 6 - Distribuição geral das pteridófitas no Estado de Pernambuco, segundo as zonas fisiográficas FAMILIAS: GLEICHENIACEAE / HYMENOPHYLLACEAE / CYATHEACEAE/ PTERIDACEAE (continuação)

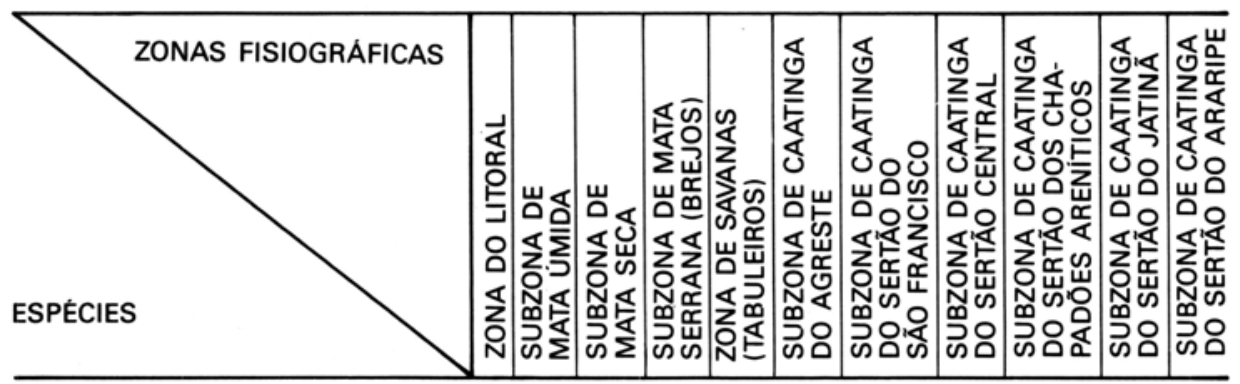

\section{GLEICHENIACEAE}

Dicranopteris pectinata (Willd.)

Underw.

Dicranopteris flexuosa (Schrad.)

Underw.

\section{HYMENOPHYLLACEAE}

Trichomanes pinnatum Hedw.

Trichomanes ovale (Fourn.) W.

Boer.

Trichomanes kraussii Hook. \& Grév.

\section{CYATHEACEAE}

Trichipteris microdonta (Desv.)

Tryon

Trichipteris procera (Willd.) Tryon

Alsophila praecincta Kze.

Cyathea delgadii Sternb.

\section{PTERIDACEAE}

Pityrograma calomelanos (L.) Link. -

Adiantopsis radiata (L.) Fée

Adiantopsis pedata (Hook.) Moore

Hemionitis tomentosa(Lam.) Raddi.

Hemionitis palmata L.

Ceratopteris thalictroides (L.)

Brong.

Ceratopteris pteridoides (HK.)

Hieron 
TABELA6 - Distribuição geral das pteridófitas no Estado de Pernambuco, segundo as zonas fisiográficas FAMILIA: PTERIDACEAE (continuação)

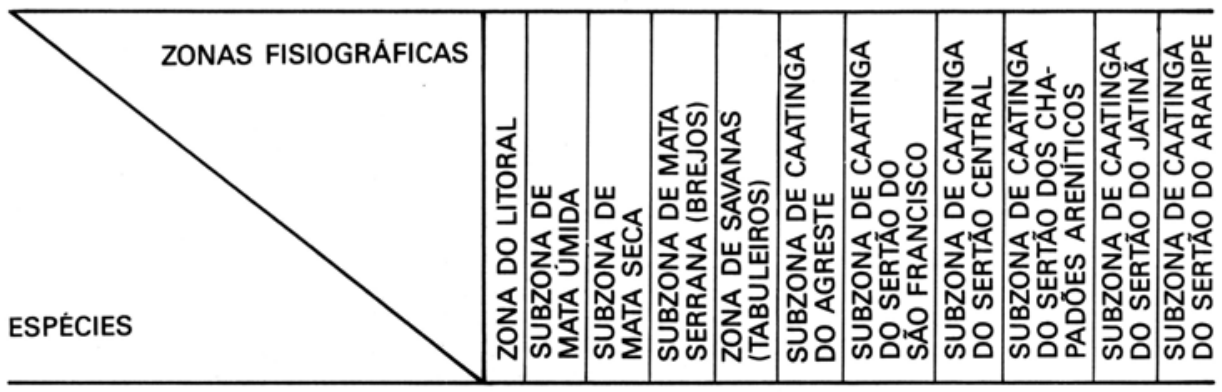

PTERIDACEAE

Cheilanthes concolor (Langsd. \&

Fisch.) R \& A. Tryon

Doryopteris varians (Raddi.) J. Sm. $-\times-{ }_{-}-{ }_{-}-{ }_{-}-{ }_{-}-$

Doryopteris collina (Raddi.) J. Sm. $-\times \quad-\quad \times \quad-\quad-\quad-\quad-\quad-\quad-\quad$

Doryopteris pedata (L.) Fée. var.

pedata Fée

Doryopteris pedata (L.) Fée. var.

multipartida (Fée.) Tryon

Doryopteris ludens (Wall.) J. Sm.

Doryopteris sagitifolia (Raddi.) J.

Sm.

Adiantum tenerum Sw.

Adiantum deflectens Mart.

Adiantum diagoanum Glaz.

Adiantum dolosum Kze.

Adiantum fovearum Raddi.

Adiantum latifolium Lam.

Adiantum intermedium $\mathrm{Sw}$.

Adiantum lunulatum Burm.

Adiantum lucidum Sw.

Adiantum serratodentatum Willd.

Adiantum tetraphyllum Willd.

Adiantum cuneatum Langsd. \&

Fisch.

Adiantum pulverulentum $\mathrm{L}$.

$$
\begin{array}{rrrrrrrrrrr}
- & - & \times & \times & - & - & - & - & - & - & - \\
- & \times & \times & \times & - & - & - & - & - & - & - \\
\hline
\end{array}
$$


TABELA 6 - Distribuição geral das Pteridófitas no Estado de Pernambuco, segundo as zonas fisiográficas FAMILIA: PTERIDACEAE (continuação)

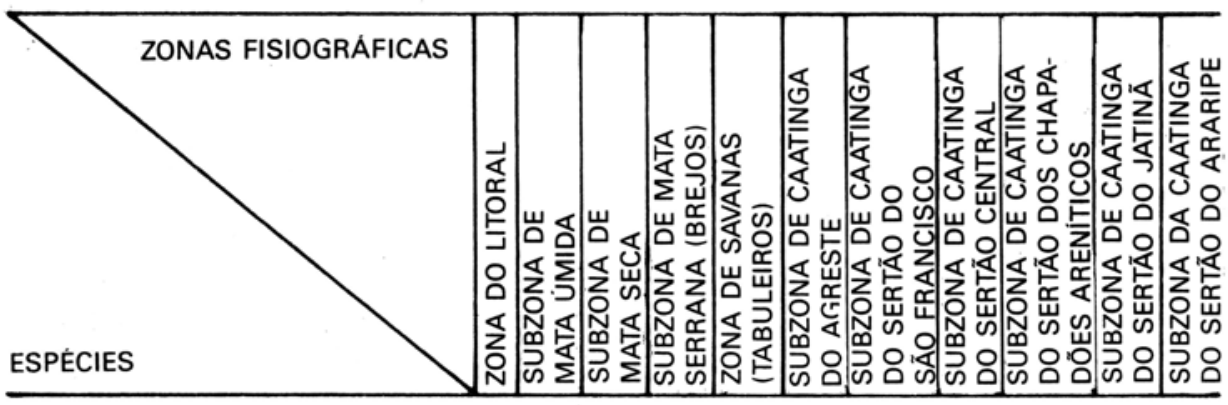

PTERIDACEAE

Adiantum caudatum L.

Adiantum sordidum Lind.

Adiantum obliquum Willd.

Adiantum villosum $\mathrm{L}$.

Adiantum terminatum Kze.

Adiantum petiolatum Desv.

Adiantum pectinatum Ettingsh.

Adiantum trapeziforme $\mathrm{L}$.

Adiantum pentadactylon Langsd.

\& Fisch.

Adiantum glaucescens $\mathrm{KI}$.

Adiantum cupillus-veneris L.

Adiantum brasiliensis Raddi.

Pteris denticulata Sw.

Pteris leptophyllum Sw.

Pteris biaurita L.

Pteris longifolia L.

Pteris vittata $\mathrm{L}$.

Pteris kunzeana Ag.

Pteris brasiliense Raddi.

Pteris dissimilis (Fée) Chr.

Acrostichum aureum $\mathrm{L}$.

Acrostichum daneaefolium

Langsd. \& Fisch.

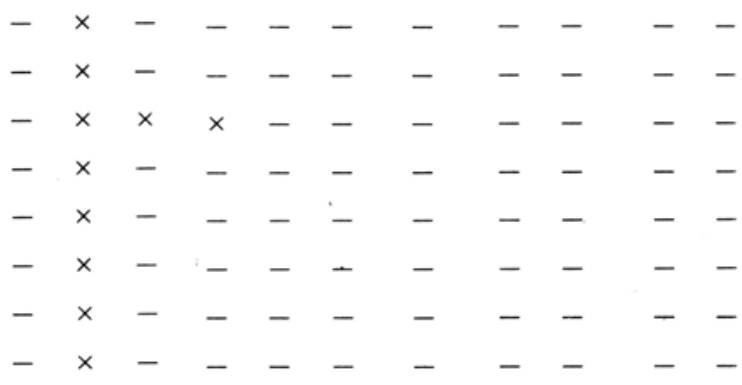


TABELA6 - Distribuição geral das pteridófitas no Estado de Pernambuco, segundo as zonas fisiográficas FAMILIAS: VITTARIACEAE/DENNSTAEDTIACEAE/THELYPTERIDACEAE/DRYOPTERIDACEAE (continuação)

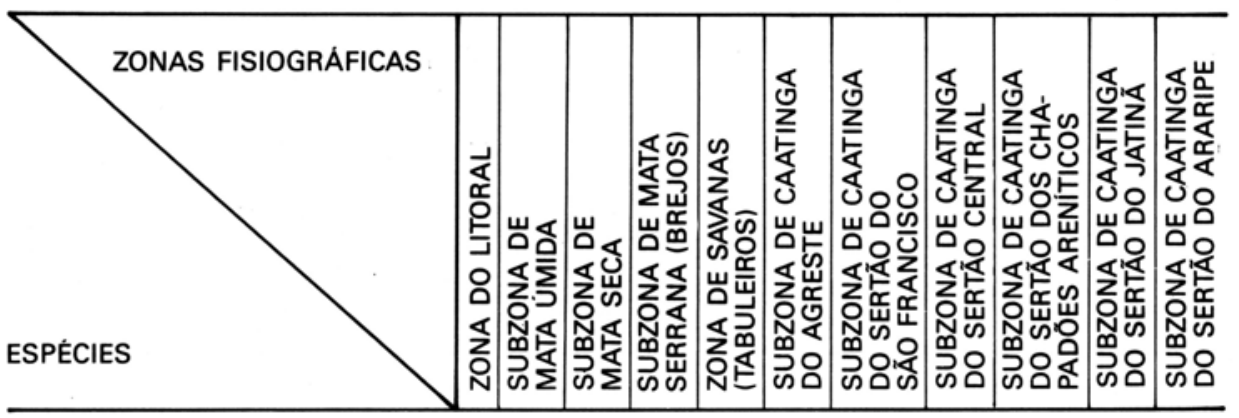

VITTARIACEAE

Vittaria lineata (L.) Sm.

Vittaria graminifolia Kaulf.

Anetium citrifolium (L.) Splitz.

Ananthacorus angustifolius (Sw.)

Underw. \& Maxon.

\section{DENNSTAEDTIACEAE}

Microlepia speluncae (L.) Moore

Saccoloma elegans Kaulf.

Pteridium aquilinum (L.) Kuhn.

Lindsaea guianensis (Aubl.) Dryand.-

Lindsaea quadriangularis Raddi.

Lindsaea stricta (Sw.) Dryand.

Lindsaea trapeziforme Dryand.

Lindsaea ovoidea Fée

Lindsaea lancea (L.) Bedd.

\section{THELYPTERIDACEAE}

Thelypteris totta (Thumb.) Schelpe -

Thelypteris dentata (Forsk.) E.P.

St. John

Thelypteris reticulata (L.) Proctor

Thelypteris serrata (Cav.) Alston DRYOPTERIDACEAE

Stigmatopteris meniscioides (Willd.).

Kramer

$\begin{array}{rrrrrrrrrrr}- & \times & - & \times & - & - & - & - & - & - \\ - & \times & - & - & - & - & - & - & - & - & -\end{array}$

$-\times \times$

$-\times-\quad-\quad-$

$-\times-\times \times-$

$\times-$

$\times-$

$-\times \times$

$-\times-$

$-\quad-$

$-\times-$ $\begin{array}{lllllllllll}- & \times & - & \times & \times & - & - & - & - & -\end{array}$

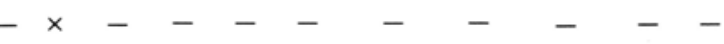

$-\times-\quad-\quad \times \quad-\quad-\quad-\quad-\quad-$ 
TABELA 6 - Distribuição geral das pteridófitas no Estado de Pernambuco, segundo as zonas fisiográficas FAMILIA: DRYOPTERIDACEAE (continuação)

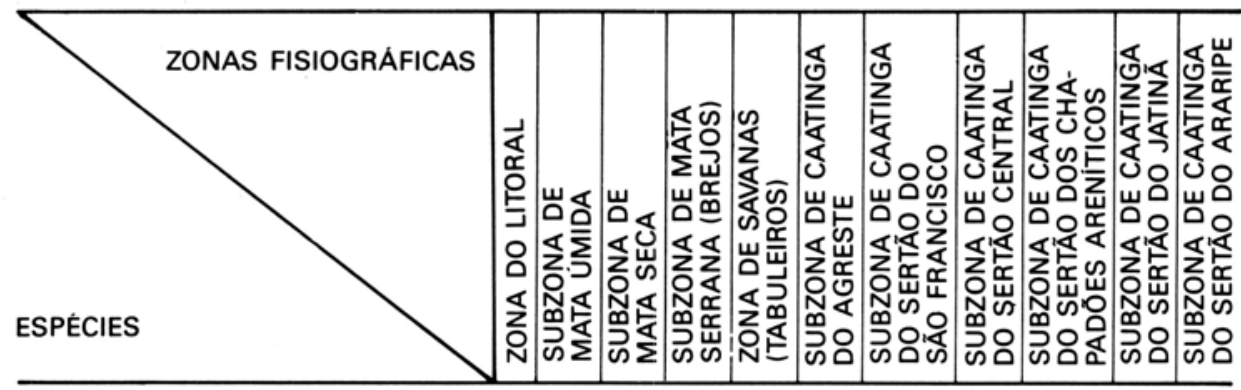

DRYOPTERIDACEAE

Tectaria heracleifolia (Willd.)

Underw.

Tectaria incisa Cav.

Rumohra adiantiformis (Forst.)

Ching

Dryopteris normalis C. Chr.

Dryopteris paucijuga C. Chr.

Dryopteris setigera (BI.) O. Ktze.

Dryopteris ctenitis (Link.) O. Ktze. -

Dryopteris alsophilaceae (Kunze)

O. Ktze.

Dryopteris mollis (Jacq.) Hieron Dryopteris effusa (Sw.) Urb.

Stigmatopteris santi-gabrielli

(Hook.) C. Chr.

Didymochlaena lunulata Desv.

Didymochlaena trunculata (Sw.)

J. Sm.

Polybotrya cervina (L.) Kep.

Polybotrya scandens (Raddi.)

Christ.

Polybotrya serratifolia (Fée) KI.

Diplazium cristatum (Desr.) Alston -

Diplazium plantaginifolium (L.)

Urban 
TABELA 6 - Distribuição geral das pteridófitas no Estado de Pernambuco, segundo as zonas fisiográficas FAMILIAS: DRYOPTERIDACEAE/ASPLENIACEAE/DAVALLIACEAE (continuação)

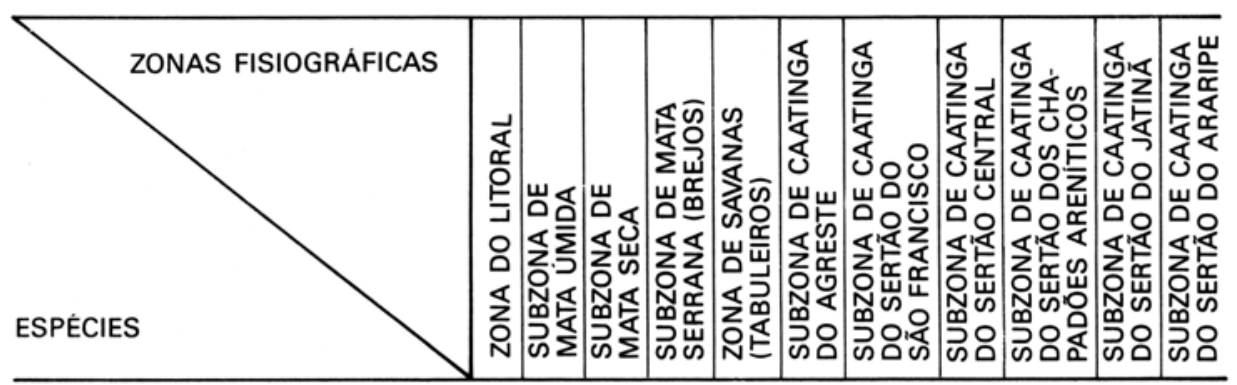

DQRYOPTERIDACEAE

Diplazium ambiguum Raddi.

Diplazium brasiliensis Ros.

Diplazium shepherdii (Spr.) Link. Lomariopsis sorbibolia (L.) Fée Lomagramma guianensis (Aubl.)

Ching

\section{ASPLENIACEAE}

Asplenium formosum Willd.

Asplenium lunulatum Sw.

Asplenium claussenii Hieron

Asplenium otites Ling.

Asplenium brachyotus Kze.

Asplenium serratum L.

Asplenium cristatum Lam.

\section{DAVALLIACEAE}

Nephrolepis exaltata (L.) Schott.

Nephrolepis pickelli Ros.

Nephrolepis pectinata (Willd.)

Schott.

Nephrolepis hirsutula (Forst.) Presl.

Nephrolepis bisserrata (Sw.)

Schott.

Nephrolepis duffii Hort.

Nephrolepis cordifolia (L.) Presl.

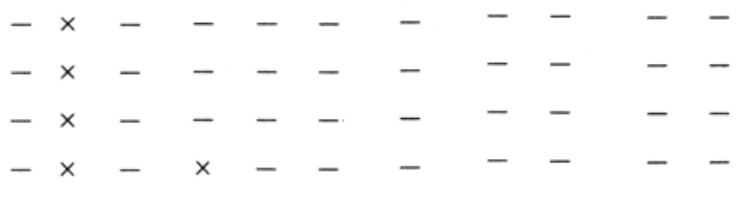


Distribuição geográfica das pteridófitas...

TABELA 6 - Distribuição geral das pteridófitas no Estado de Pernambuco, segundo as zonas fisiográficas FAMILIAS: BLECHNACEAE, POLYPODIACEAE (continuação)

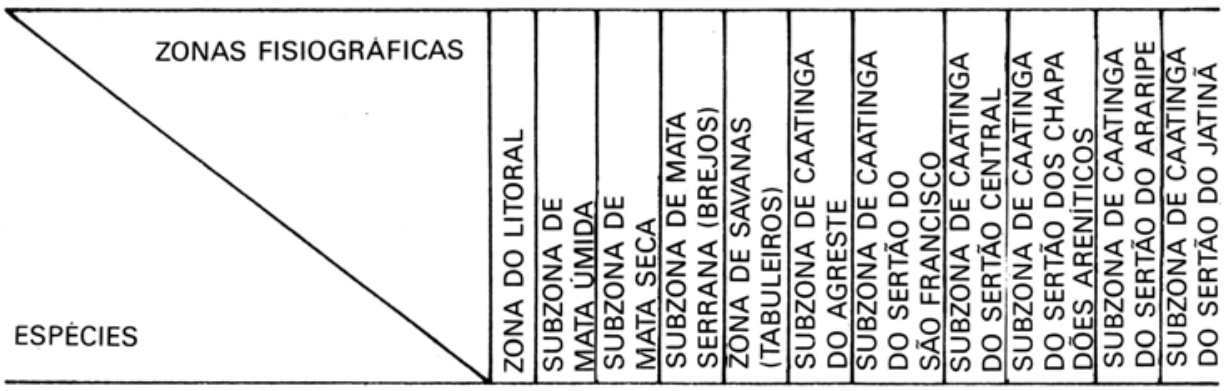

\section{BLECHNACEAE}

Blechnum occidentale L.

Blechnum brasiliense Desv.

Blechnum serrulatum L.C. Rich.

Blechnum volubilis (KIf.) Hook. POLYPODIACEAE

Dicranoglossum furcatum (L.) J.

Sm.

Dicranoglossum desvauxii (KI.)

Proctor

Microgramma vaccinifolia (Langsd.

\& Fisch.) Copel.

Microgramma geminata (Schrad.)

R. \& A. Tryon

Pleopeltis macrocarpa (Willd.)

Kaulf.

Polypodium brasiliense Poir.

Polypodium aureum L.

Polypodium decumanum Willd.

Polypodium hirsutissimum Raddi.

Polypodium catharinae Langsd. \&

Fisch.

Polypodium lapathifolium Poiret

Polypodium escorpioides L.

Polypodium stenophyllum BI.

$\begin{array}{ccccccccccc}- & \times & \times & \times & - & \times & - & - & \times & - & - \\ - & \times & \times & \times & - & - & - & - & - & - & - \\ \times & \times & - & - & \times & - & - & - & - & - & - \\ - & \times & - & - & - & - & - & - & - & - & -\end{array}$
$-\times-\times-$ 
TABELA 6 - Distribuição geral das pteridófitas no Estado de Pernambuco, segundo as zonas fisiográficas FAMILIA: POLYPODIACEAE (continuação)

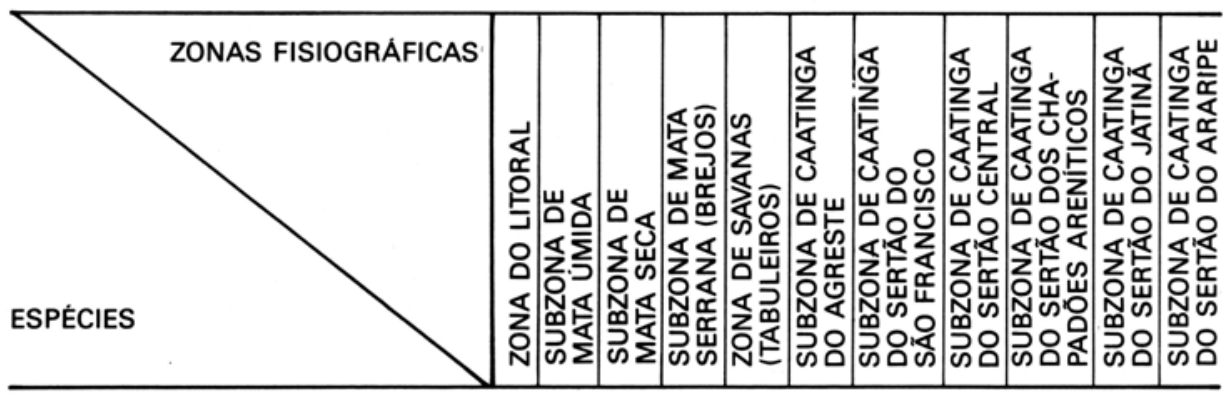

POLYPODIACEAE

Polypodium lineatum Colebr.

Polypodium lineare Thbg.

Polypodium lycopodioides L.

Pleopeltis revoluta (Willd.) A.R.

Sm.

Polypodium paradiseae Langsd. \&

Fisch.

Polypodium fraxinifolium Jacq.

Polypodium crassifolium L.

Polypodium areolatum H.B.W.

Polypodium laevigatum Cav.

Polypodium pectinatum $\mathrm{L}$.

Polypodium pectinatiforme Lind.

Polypodium phyllitidis L.

Polypodium landigii L.

Polypodium lepidopteris (Langsd.

घे Fisch.) Kze.

Polypodium glofium L.

Polypodium polypodioides (L.)

Watt.

Polypodium apiculatum Kze.

Polypodium angustum (H.B.W.)

Liebm.

Campyloneurum repens (Aubl.)

Presl.

$$
\begin{aligned}
& -\quad-\quad \times \quad-\quad-\quad-\quad-\quad-\quad-
\end{aligned}
$$

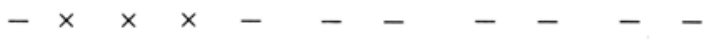

$$
\begin{aligned}
& -\times-\quad-\quad-\quad \times \quad-\quad-\quad-\quad-\quad- \\
& -\times-\times-\infty--\infty \\
& -\times-\quad-\quad-\quad-\quad-\quad-\quad-\quad- \\
& -\times--\quad-\quad-\quad-\quad-\quad-\quad- \\
& -\times---\infty-\infty-- \\
& -\quad \times \quad-\quad-\quad-\quad-\quad-\quad-\quad- \\
& -\quad-\times--\quad-\quad-\quad-\quad-\quad- \\
& -\quad-\quad-\quad \times \quad-\quad-\quad-\quad-\quad-\quad-1 \\
& -\quad-\quad \times-\quad-\quad-\quad-\quad-\quad-
\end{aligned}
$$

Prest. 
TABELA 6 - Distribuição geral das pteridófitas no Estado de Pernambuco, segundo as zonas fisiográficas FAMILIAS: POLYPODIACEAE/MARSILEACEAE/SALVINIACEAE/PSILOTACEAE/LYCOPODIACEAE

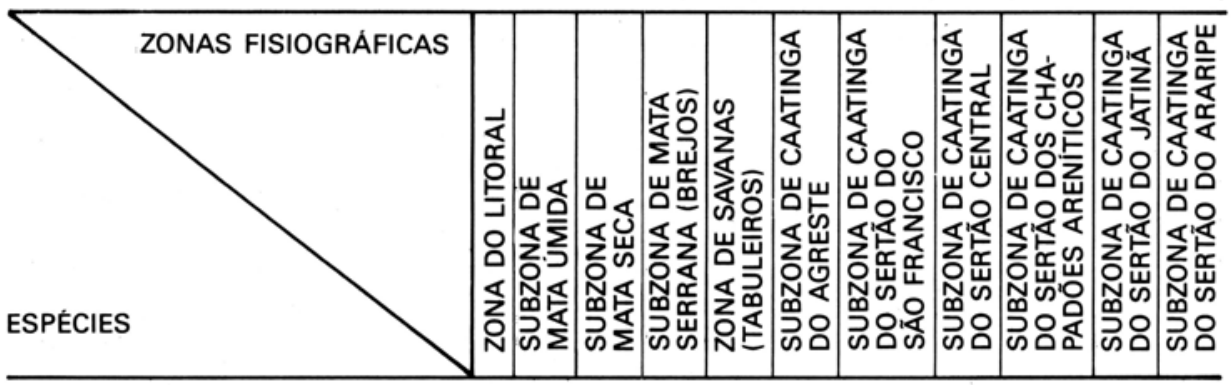

POLYPODIACEAE

Campyloneurum transparens Ett.

Campyloneurum angustifolium

(Sw.) Fée

Cochlidium paucinervatum (Fée)

C. Chr.

MARSILEACEAE

Marsilea deflexa A. Braum.

Marsilea quadrifolia L.

\section{SALVINIACEAE}

Salvinia auriculata Aubl.

Azolla caroliniana Willd.

Azolla microphylla Kaulf.

\section{PSILOTACEAE}

Psilotum nudum (L.) Beauv. LYCOPODIACEAE

Lycopodium cernuum L.

Lycopodium heterocarpon Fée

Lycopodium carolinianum L.

Lycopodium taxifolium L.

Lycopodium setaceus (Hamilt.)

Hert.

Lycopodium christii Alv. Silv.

Lycopodium alopecuroides $\mathrm{L}$.

Lycopodium treitubensis Alv. Silv. - 
TABELA 6 - Distribuição geral das pteridófitas no Estado de Pernambuco, segundo as zonas fisiográficas FAMILIAS: LYCOPODIACEAE, SELAGINELLACEAE (conclusão)

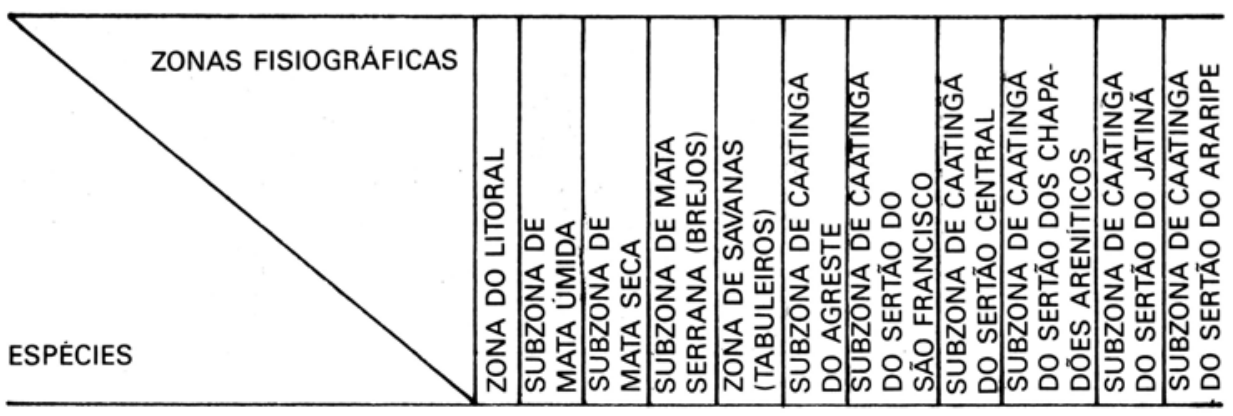

\section{LYCOPODIACEAE}

Lycopodium linifolium L.

Lycopodium regnellii Maxon.

Lycopodium martii Alv. Silv.

\section{SELAGINELLACEAE}

Selaginella convoluta (Arn.)

Spring.

Selaginella breynei Spring. ex Char.

Selaginella willdenowii (Desv.)

Baker

Selaginella simplex Baker

Selaginella muscova $\mathrm{A}$. Br.

Selaginella flagellata Spring.

Selaginella deltoidea $\mathrm{A}$. Br.

Selaginella fragillima A. Silv.

Selaginella vallida Alston

$\begin{array}{ccccccccccc}- & \times & - & - & - & - & - & - & - & - & - \\ - & \times & - & - & - & - & - & - & - & - & - \\ - & \times & - & - & - & - & - & - & - & - & - \\ - & \times & - & - & - & - & - & - & - & - & - \\ - & \times & - & - & - & - & - & - & - & - & - \\ - & \times & - & - & - & - & - & - & - & - & - \\ - & \times & - & - & - & - & - & - & - & - & - \\ - & - & - & - & - & - & - & \times & - & - & -\end{array}$

Selaginella seilowii Hieron 
convivem com essas áreas. Tem-se desta forma a ocorrência das Salviniaceae, Marsileaceae e Selaginellaceae na subzona do sertão do Araripe.

Para uma visualização global da ocorrência das pteridófitas no Estado de Pernambuco, vide a Tabela 6, na qual se evidencia a totalidade das espécies estudadas segundo as zonas e subzonas fisiográficas.

\section{Conclusões}

- As pteridófitas ocorrem em todas as zonas fisiográficas do Estado de Pernambuco.

- Estão representadas pelas famílias Ophioglossaceae, Marattiaceae, Schizaeaceae, Gleicheniaceae, Hymenophyllaceae, Cyatheaceae, Pteridaceae, Vittariaceae, Dennstaedtiaceae, Thelypteridaceae, Dryopteridaceae, Aspleniaceae, Davalliaceae, Blechnaceae, Polypodiaceae, Marsileaceae, Salviniaceae, Psilotaceae, Lycopodiaceae e Sellaginellaceae (sistema de Tryon \& Tryon, 1982).

- São mais bem representadas quanto ao número de espécies as Pteridaceae, as Dryopteridaceae e as Polypodiaceae.

- A zona fisiográfica com maior número de espécies é a de mata, especialmente a subzona de mata úmida.

- Os dados apresentados indicam uma provável ocorrência de uma flora pteridofítica diferenciada nas matas serranas ou brejos de altitude.

- As Schizaeaceae, através do gênero Anemia Swartz., e as Sellaginellaceae, através do gênero Selaginella Palisot de Beauvois, demonstram um potencial de adaptação e resistência a ambientes xéricos muito forte, com a ocorrência de espécies com dispersão desde áreas úmidas de mata até zonas de caatinga do sertão.

- A presença das Hydropteridinae nas zonas e subzonas de caatinga do Estado, ocorrendo em "'estanques d'água" na época chuvosa, sugere-nos um provável estágio das espécies, no período de seca, sob a forma de esporocarpos de "resistência",

\section{Agradecimentos}

$\mathrm{Na}$ conclusão deste trabalho, agradecemos a todas as pessoas que colaboraram para a sua execução, destacando:

O Dr. Geraldo Mariz, pelos incentivos e colaboração prestados.

A Dra Laise de Holanda Cavalcanti-Andrade, pelas idéias e ajudas sugeridas.

O Dr. Paulo Gunther Windisch, pelos incentivos e sugestões dados.

A bióloga Ana Maria da Silva Correia, pela redação do Abstract.

O estagiário Fábio Henrique do Couto Soares, pelas contribuições prestadas.

Os curadores dos herbários IPA, PEUFR, HST e UFP, pelo empréstimo de materiais. 


\section{Referências bibliográficas}

ANDRADE-LIMA, D. de. 1950. Catálogo do Herbário da Escola de Agricultura em Tapera, Pernambuco. Boletim do S.A.I.C. de Pernambuco, 1(2): 68-126. 1960. Estudos Fitogeográficos de Pernambuco. Arquivos do Instituto de Pesquisas Agronômicas. 2a ed. Vol. (5): 305-341.

1961. Tipos de Florestas de Pernambuco. São Paulo, Anais da Associação dos Geógrafos Brasileiros.

1969. Pteridófitas que ocorrem nas Floras Extra-Amazônicas do Brasil e proximidades. In: Anais da Sociedade Botânica do Brasil. 20., Goiânia.

ARENS, K. \& POTSCH, S. 1969. Sobre a Ecologia da Selaginella sellowii Hieron. Lilloa, 20: $89-104$.

BARROS, I.C.L. 1980. Taxonomia, Fitogeografia e Morfologia das Schizaeaceae do Nordeste Brasileiro. Dissertação de Mestrado. UFRPE. Recife, Pernambuco.

1982. Biotaxonomia das Espécies de Anemia ocorrentes no Nordeste brasileiro. Uma contribuição ao estudo das Schizaeaceae do Brasil. In: Resumos do XXXIII Congresso Nacional de Botânica, Maceió.

1982. Estudos Biotaxonômicos das Espécies de Schizaea e Lygodium ocorrentes no Nordeste brasileiro. Uma contribuição do estudo das Schizaeaceae do Brasil. In: Resumos do XXXIII Congresso Nacional de Botânica, Maceió.

BARROS, I.C.L. \& MARIZ, G. 1985. Novas Referências de Pteridófitas para Pernambuco (BR). In. Resumos do XXXVI Congresso Nacional de Botânica, Curitiba. (Entregue para publicação.)

BARROS, I.C.L.; SILVA, A. de J. R. da \& SOARES, F.H. do C. 1986. Novas referências de Pteridófitas para Pernambuco. II. PTERIDACEAE. DENNSTAEDTIACEAE. THELYPTERIDACEAE. DRYOPTERIDACEAE. In: Congresso Nacional de Botânica. 37.', Ouro Preto. (Entregue para publicação.)

1986. Novas Referências de Pteridófitas para Pernambuco. III. ASPLENIACEAE. DAVALLIACEAE. POLYPODIACEAE. MARSILEACEAE. LYCOPODIACEAE. In: Congresso Nacional de Botânica. 37., Ouro Preto. (Entregue para publicação.)

BARROS, I.C.L.; SILVA, A. de J. R. \& ANDRADE, L. de H.C. 1985. Distribuição das Espécies de Pteridófitas Ocorrentes na Mata de Dois Irmãos (Recife-PE). In: Reunião Nordestina de Botânica. 9., Aracaju.

1986. Nova Referência de Pteridófita em Área Remanescente da Floresta Atlântica (Mata de Dois Irmão-Recife-PE). In: Congresso Nacional de Botânica. 37.̊, Ouro Preto. (Entregue para publicação.)

CHRISTENSEN, C. 1973. Index Filicum (1753-1905). Reprint by Otto Koeltz Antiquariat. CHRISTENSEN, C. 1973. Index Filicum (1906-1933). Reprint by Otto Koeltz Antiquariat. Suplementum I-III.

De LA SOTA, E.R. 1966. Sobre la Presencia de la Selaginella convoluta (Walk. Arn.) Spring. en Argentina. Boletin de la Sociedad Argentina de Botánica, 11(1): 39-41.

PICHI-SERMOLLI, R.E.G. 1965. Index Filicum. Pro Annis 1934-1960. Suplementum Quartum. UNESCO-IUBS.

PONTUAL, I.B. 1969. Pteridófitas de Pernambuco e Alagoas (II). In: Anais do Congresso Nacional de Botânica. 20, Goiânia. 
1971. Pteridófitas de Pernambuco e Alagoas (I). In: Anais do Instituto de Ciências Biológicas da Universidade Federal Rural de Pernambuco, Recife, 1(1): 153-260.

1972. Pteridófitas do Nordeste. In: Anais do Congresso Nacional de Botânica. 23., Garanhuns.

SARMENTO, A.C. 1960. Fitofisionomia da Lagoa de Pau-Sangue. Arquivos do Instituto de Pesquisas Agronômicas de Pernambuco, 5: 223-257.

SEHNEM, A. 1968. Blechnaceas. Flora Ilustrada Catarinense. Fasc. BLEC, Itajai: 1-90. 1968. Aspleniaceas. Flora Ilustrada Catarinense. Fasc. ASPL, Itajaí: 1-96. 1970. Polipodiaceas. Flora Ilustrada Catarinense. Fasc. POLI. Itajaí: 1-173. 1971. Himenofilaceas. Flora Ilustrada Catarinense. Fasc. HIME, Itajaí: 1-98. 1972. Pteridaceas. Flora Ilustrada Catarinense. Fasc. PTER, Itajaí: 1-244. 1974. Esquizeaceas. Flora Ilustrada Catarinense. Fasc. ESQUI, Itajaí: 1-78. 1974. Davaliaceas. Flora Ilustrada Catarinense. Fasc. DAVA, Itajaí: 1-18.

SILVA, G.C. da. 1985. Flora de Vegetação das Depressões Inundáveis da Região de OuricuriPE. Dissertação de Mestrado, UFRPE, Recife-PE.

STRITTMATER, G.G.D. de. 1973. Nueva Técnica de Diafanización. Buenos Aires, Bolletín de la Sociedad Argentina de Botánica, 15(1): 126-129.

TRYON, R.M. \& CONANT, D.S. 1975. The Ferns of Brazilian Amazonia. Acta Amazonica, 5(1): 23-34.

TRYON \& TRYON, A.F. 1982. Ferns and Allied Plants with Special Reference to Tropical America. New York, Springer-Verlag. 

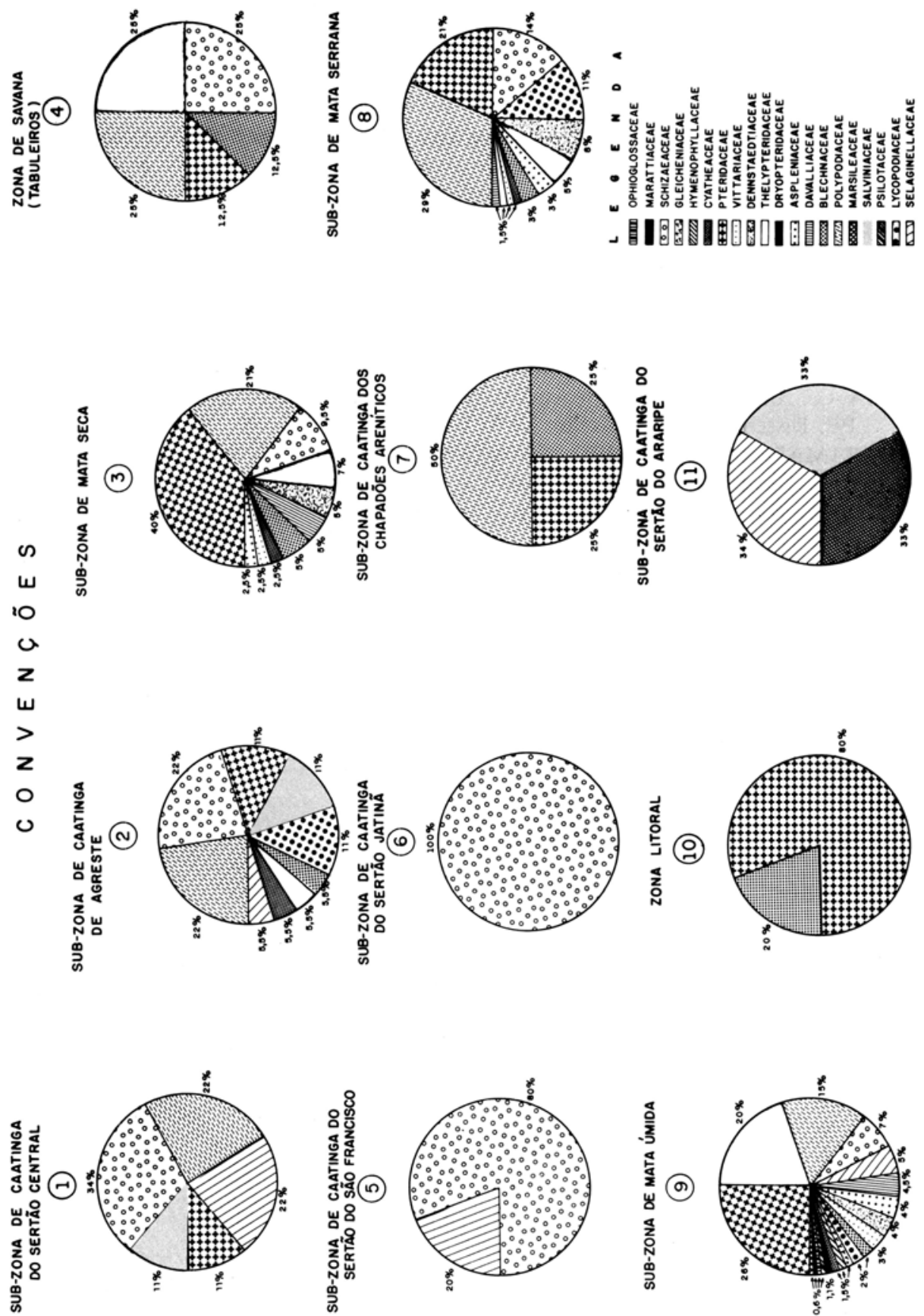

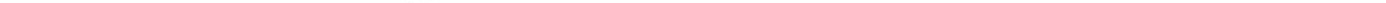